\title{
Mathematical Models of the Homochiralisation of Crystals by Grinding
}

\author{
Jonathan A. D. Wattis
}

Received: 7 October 2009 / Accepted: 19 July 2010 /

Published online: 4 August 2010

(C) The Author(s) 2010. This article is published with open access at Springerlink.com

\begin{abstract}
We review the existing mathematical models which describe physicochemical mechanisms capable of producing a symmetry-breaking transition to a state in which one chirality dominates the other. A new model is proposed, with the aim of elucidating the fundamental processes at work in the crystal grinding systems of Viedma (Phys Rev Lett 94:065504, 2005) and Noorduin (J Am Chem Soc 130:1158-1159, 2008). We simplify the model as far as possible to uncover the fundamental competitive process which causes the symmetry-breaking, and analyse other simplifications which might be expected to show symmetry-breaking.
\end{abstract}

Keywords Symmetry-breaking $\cdot$ Homochirality $\cdot$ Crystal growth . Enantiomeric excess $\cdot$ Mathematical modelling

\section{Introduction}

A significant stage in the formation of living systems was the transition from a symmetric chemistry involving mirror-symmetric and approximately equal numbers of left- and right-handed chiral species into a system involving just one-handedness of chiral molecules.

In this paper we focus on mathematical models of one example of a physicochemical system which undergoes such a symmetry-breaking transition, namely the crystal grinding processes investigated by Viedma (2005) and Noorduin et al. (2008), which have been recently reviewed by McBride and Tully (2008). Our aim is to

\section{J. A. D. Wattis $(\bowtie)$}

Theoretical Mechanics, School of Mathematical Sciences,

University of Nottingham, University Park, Nottingham NG7 2RD, UK

e-mail: Jonathan.Wattis@nottingham.ac.uk 
describe this process by way of a detailed microscopic model of the nucleation and growth processes and then to simplify the model, retaining only the bare essential mechanisms responsible for the symmetry-breaking bifurcation.

We start by reviewing the processes which are already known to cause a symmetrybreaking bifurcation. By this we mean that a system which starts off in a racemic state (one in which both left-handed and right-handed structures occur with approximately equal frequencies) and, as the system evolves, the two handednesses grow differently, so that at a later time, one handedness is predominant in the system.

\section{Models for Homochiralisation}

Many models have been proposed for the emergence of homochirality from an initially racemic mixture of precursors. Frank (1953) proposed an open system into which $R$ and $S$ particles are continually introduced, and combine to form one of two possible products: left- or right-handed species, $X, Y$. Each of these products acts as a catalyst for its own production (autocatalysis), and each combines with the opposing handed product (cross-inhibition) to form an inert product $(P)$ which is removed from the system at some rate. These processes are summarised by the following reaction scheme:

$$
\begin{aligned}
& \text { external source } \rightarrow R, S \text { input, } k_{0}, \\
& R+S \rightleftharpoons X \quad R+S \rightleftharpoons Y \quad \text { slow, } k_{1}, \\
& R+S+X \rightleftharpoons 2 X \quad R+S+Y \rightleftharpoons 2 Y \quad \text { fast, autocatalytic, } k_{2} \\
& X+Y \rightarrow P \quad \text { cross-inhibition, } k_{3} \text {, } \\
& P \rightarrow \quad \text { removal, } k_{4} \text {. }
\end{aligned}
$$

Ignoring the reversible reactions (for simplicity), this system can be modelled by the differential equations

$$
\begin{aligned}
& \frac{\mathrm{d} r}{\mathrm{~d} t}=k_{0}-2 k_{1} r s-k_{2} r s(x+y)+k_{-1}(x+y)+k_{-2}\left(x^{2}+y^{2}\right), \\
& \frac{\mathrm{d} s}{\mathrm{~d} t}=k_{0}-2 k_{1} r s-k_{2} r s(x+y)+k_{-1}(x+y)+k_{-2}\left(x^{2}+y^{2}\right), \\
& \frac{\mathrm{d} x}{\mathrm{~d} t}=k_{1} r s+k_{2} r s x-k_{3} x y-k_{-1} x-k_{-2} x^{2}, \\
& \frac{\mathrm{d} y}{\mathrm{~d} t}=k_{1} r s+k_{2} r s y-k_{3} x y-k_{-1} y-k_{-2} y^{2}, \\
& \frac{\mathrm{d} p}{\mathrm{~d} t}=k_{3} x y-k_{4} p,
\end{aligned}
$$

from which we note that at steady-state we have

$$
r s=\frac{k_{0}+k_{-1}(x+y)+k_{-1}\left(x^{2}+y^{2}\right)}{2 k_{1}+k_{2}(x+y)} .
$$


We write the absolute enantiomeric excess as $e e=x-y$ and the total concentration as $\sigma=x+y$; adding and subtracting the equations for $\mathrm{d} x / \mathrm{d} t$ and $\mathrm{d} y / \mathrm{d} t$, we find

$$
\begin{gathered}
\sigma^{2}=\frac{2 k_{0}}{k_{3}}+e e^{2}, \\
e e\left[\frac{k_{2}\left(k_{-2} e e^{2}+k_{-2} \sigma^{2}+2 k_{-1} \sigma+2 k_{0}\right)}{2\left(2 k_{1}+k_{2} \sigma\right)}-k_{-1}-k_{-2} \sigma\right]=0 .
\end{gathered}
$$

Hence $e e=0$ is always a solution, and there are other solutions with $e e \neq 0$ if the rate constants $k_{*}$ satisfy certain conditions (these include $k_{3}>k_{-2}$ and $k_{0}$ being sufficiently large).

The important issues to note here are:

(i) this system is open, it requires the continual supply of fresh $R, S$ to maintain the asymmetric steady-state. Also, the removal of products is required to avoid the input terms causing the total amount of material to increase indefinitely;

(ii) the forcing input term drives the system away from an equilibrium solution, into a distinct steady-state solution;

(iii) the system has cross-inhibition which removes equal numbers of $X$ and $Y$, amplifying any differences caused by random fluctuations in the initial data or in the input rates.

Saito and Hyuga (2004) discuss a sequence of toy models describing homochirality caused by nonlinear autocatalysis and recycling. Their family of models can be summarised by

$$
\begin{aligned}
& \frac{\mathrm{d} r}{\mathrm{~d} t}=k r^{2}(1-r-s)-\lambda r, \\
& \frac{\mathrm{d} s}{\mathrm{~d} t}=k s^{2}(1-r-s)-\lambda s,
\end{aligned}
$$

where $r$ and $s$ are the concentrations of the two enantiomers. Initially they consider $k_{r}=k_{s}=k$ and $\lambda=0$ and find that enantiomeric exess, $r-s$ is constant. Next the case $k_{r}=k r, k_{s}=k s, \lambda=0$ is analysed, wherein the relative enantiomeric excess $\frac{r-s}{r+s}$ is constant. Then the more complex case of $k_{r}=k r^{2}, k_{s}=k s^{2}, \lambda=0$ is analysed, and amplification of the enantiomeric excess is obtained. This amplification persists when the case $\lambda>0$ is finally analysed. This shows us strong autocatalysis may cause homochiralisation, but in any given experiment, it is not clear which form of rate coefficients $\left(k_{r}, k_{s}, \lambda\right)$ should be used.

Saito and Hyuga (2005) analyse a series of models of crystallisation which include some of features present in our more general model. They note that a model truncated at tetramers exhibits different behaviour from one truncated at hexamers. In particular, the symmetry-breaking phenomena is not present in the tetramer model, but is exhibited by the hexamer model. Hence, later, we will consider models 
truncated at the tetramer and the hexamer levels and investigate the differences in symmetry-breaking behaviour ("The Truncation at Tetramers" and "The Truncation at Hexamers").

Denoting monomers by $c$, small and large left-handed clusters by $x_{1}, x_{2}$ respectively and right-handed by $y_{1}, y_{2}$, Uwaha (2004) writes down the scheme

$$
\begin{aligned}
& \frac{\mathrm{d} c}{\mathrm{~d} t}=-2 k_{0} c^{2}-k_{1} c\left(x_{1}+y_{1}\right)+\lambda_{1}\left(x_{2}+y_{2}\right)+\lambda_{0}\left(x_{1}+y_{1}\right), \\
& \frac{\mathrm{d} x_{1}}{\mathrm{~d} t}=k_{0} c^{2}-k_{u} x_{1} x_{2}-k_{c} x_{1}^{2}+\lambda_{u} x_{2}+\lambda_{0} x_{1}, \\
& \frac{\mathrm{d} x_{2}}{\mathrm{~d} t}=k_{1} x_{2} c+k_{u} x_{1} x_{2}+k_{c} x_{1}^{2}-\lambda_{1} x_{2}-\lambda_{u} x_{2}, \\
& \frac{\mathrm{d} y_{1}}{\mathrm{~d} t}=k_{0} c^{2}-k_{u} y_{1} y_{2}-k_{c} y_{1}^{2}+\lambda_{u} y_{2}+\lambda_{0} y_{1}, \\
& \frac{\mathrm{d} y_{2}}{\mathrm{~d} t}=k_{1} y_{2} c+k_{u} y_{1} y_{2}+k_{c} y_{1}^{2}-\lambda_{1} y_{2}-\lambda_{u} y_{2},
\end{aligned}
$$

which models

- the formation of small chiral clusters $\left(x_{1}, y_{1}\right)$ from an achiral monomer $(c)$ at rate $k_{0}$,

- small chiral clusters $\left(x_{1}, y_{1}\right)$ of the same handedness combining to form larger chiral clusters (rate $k_{c}$ ),

- $\quad$ small and larger clusters combining to form larger clusters (rate $k_{u}$ ),

- large clusters combining with achiral monomers to form more large clusters at the rate $k_{1}$,

- the break up of larger clusters into smaller clusters (rate $\lambda_{u}$ ),

- the break up of small clusters into achiral monomers (rate $\left.\lambda_{0}\right)$,

- the break up of larger clusters into achiral monomers (rate $\left.\lambda_{1}\right)$.

Such a model can exhibit symmetry-breaking to a solution in which $x_{1} \neq x_{2}$ and $x_{2} \neq y_{2}$. Uwaha points out that the recycling part of the model (the $\lambda_{*}$ parameters) are crucial to the formation of a 'completely' homochiral state. One problem with such a model is that since the variables are all total masses in the system, the size of clusters is not explicitly included. This can easily be overcome by using a more formal coarse-grained model such as that of Bolton and Wattis (2003). In asymmetric distributions, the typical size of left- and right- handed clusters may differ drastically, hence the rates of reactions will proceed differently in the cases of a few large crystals or many smaller crystals.

Sandars has proposed a model of symmetry-breaking in the formation of chiral polymers (2003). His model has an achiral substrate $(S)$ which splits into chiral monomers $L_{1}, R_{1}$ both spontaneously at a slow rate and at a faster rate, when catalysed by the presence of long homochiral chains. This catalytic effect has both autocatalytic and crosscatalytic components, that is, for example, the presence of long right-handed chains $R_{n}$ autocatalyses the production of right-handed monomers $R_{1}$ from $S$, (autocatalysis) as well as the production of left-handed monomers, $L_{1}$ (crosscatalysis). Sandars assumes the growth rates of chains are linear and not 
catalysed; the other mechanism required to produce a symmetry-breaking bifurcation to a chiral state is cross-inhibition, by which chains of opposite handednesses interact and prevent either from further growth. These mechanisms are summarised by

$$
\begin{aligned}
& S \rightarrow L_{1}, \quad S \rightarrow R_{1}, \quad \text { slow, } \\
& S+L_{n} \rightarrow L_{1}+L_{n}, S+R_{n} \rightarrow R_{1}+R_{n}, \quad \text { autocatalytic, rate } \propto 1+f, \\
& S+R_{n} \rightarrow L_{1}+R_{n}, S+L_{n} \rightarrow R_{1}+L_{n}, \quad \text { cross-catalytic, rate } \propto 1-f, \\
& L_{n}+L_{1} \rightarrow L_{n+1}, \quad R_{n}+R_{1} \rightarrow R_{n+1}, \quad \text { chain growth, rate }=a, \\
& L_{n}+R_{1} \rightarrow Q_{n+1}, \quad R_{n}+L_{1} \rightarrow P_{n+1}, \quad \text { cross-inhibition, rate }=a \chi \text {. }
\end{aligned}
$$

This model and generalisations of it have been analysed by Sandars (2003), Brandenburg et al. (2005a, b), Multimaki and Brandenburg (2005), Wattis and Coveney (2005a, b), Gleiser and Walker (2008), Gleiser et al. (2008), Coveney and Wattis (2006). Typically a classic pitchfork bifurcation is found when the fidelity $(f)$ of the autocatalysis over the cross-catalysis is increased. One counterintuitive effect is that increasing the cross-inhibition effect $(\chi)$ aids the bifurcation, allowing it to occur at lower values of the fidelity parameter $f$.

\section{Experimental Results on Homochiralisation}

The Soai reaction was one of the first experiments which demonstrated that a chemical reaction could amplify initial small imbalances in chiral balance; that is, a small enantiomeric exess in catalyst at the start of the experiment led to a much larger imbalance in the chiralities of the products at the end of the reaction. Soai et al. (1995) was able to achieve an enantiomeric exess exceeding $85 \%$ in the asymmetric autocatalysis of chiral pyrimidyl alkanol.

The first work showing that crystallisation experiments could exhibit symmetry breaking was that of Kondepudi and Nelson (1990). Later Kondepudi et al. (1995) showed that the stirring rate was a good bifurcation parameter to analyse the final distribution of chiralities of crystals emerging from a supersaturated solution of sodium chlorate. With no stirring, there were approximately equal numbers of leftand right-handed crystals. Above a critical (threshold) stirring rate, the imbalance in the numbers of each handedness increased, until, at large enough stirring rates, total chiral purity was achieved. This is due to all crystals in the system being derived from the same 'mother' crystal, which is the first crystal to become established in the system; all other crystals grow from fragments removed from it (either directly or indirectly). Before this, Kondepudi and Nelson (1984, 1985) worked on the theory of chiral symmetry-breaking mechanisms with the aim of predicting how parity-violating perturbations could be amplified to give an enantiomeric exess in prebiotic chemistry, and the timescales involved. Their results suggest a timescale of approximately $10^{4}$ years. More recently, Kondepudi and Asakura (2001) have summarised both the experimental and theoretical aspects of this work.

Viedma (2005) was the first to observe that grinding a mixture of chiral crystals eventually led to a distribution of crystals which were all of the same handedness. The 
crystalline material used was sodium chlorate, as used by Kondepudi et al. (1990). Samples of L and D crystals are mixed with water in round-bottomed flasks and the system is stirred by a magnetic bar (of length 3-20mm) at $600 \mathrm{rpm}$. The system is maintained in a supersaturated state; small glass balls are added to continually crush the crystals. The grinding is thus continuous, and crystals are maintained below a size of $200 \mu \mathrm{m}$. The chirality of the resulting crystals was determined by removing them from the flask, allowing them to grow and measuring their optical activity. The results show that, over time, the percentages of left- and right-handed crystals steadily change from about $50 / 50$ to $100 / 0$ or $0 / 100$ - a state which is described as complete chiral purity. With stirring only and no glass balls, the systems conserve their initial chiral excesses; with glass balls present and stirring, the chiral excess increases, and this occurs more rapidly if more balls are present or the speed of stirring is increased.

More recently, Noorduin et al. (2008) have observed a similar effect with amino acids - a much more relevant molecule in the study of origins of life. This work has been reviewed by McBride and Tully (2008), who add to the speculation on the mechanisms responsible for the phenomenon. Noorduin et al. describe grinding as 'dynamic dissolution/crystallization processes that result in the conversion of one solid enantiomorph into the other'. They also note that 'once a state of single chirality is achieved, the system is "locked" because primary nucleation to form and sustain new crystals from the opposite enantiomer is kinetically prohibited'. Both these quotes include the crucial fact that the process evolves not towards an equilibrium solution (which would be racemic), but towards a different, dynamic steady-state solution. As noted by Plasson (personal communication, 2008), this nonequilibrium state is maintained due to the constant input of energy into the system through the grinding process.

McBride and Tully (2008) discuss the growth of one enantiomorph, and the dissolution of the other as a type of Ostwald ripening process; with the large surface area to volume ratio of smaller crystals giving a rapid dissolution rate, whilst larger crystals, have a lower surface area to volume ratio meaning that they dissolve more slowly. However appealing such an argument maybe, since surface area arguments can equally well be applied to the growth side of the process, it is not clear that this is either necessary or sufficient. Infact, the model analysed later in this paper will show that a critical cluster size is not necessary to explain homochiralisation through grinding.

\section{Our Aims}

We aim to describe the results of the crystal grinding phenomenon through a model which recycles mass through grinding, which causes crystals to fragment, rather than having explicit mass input and removal. Simultaneously we need crystal growth processes to maintain a distribution of sizeable crystals.

We assume that the crystals are solids formed in an aqueous environment, however, we leave open questions as to whether they are crystals of some mineral of direct biological relevance (such as amino acids), or whether they are some other material, which after growing, will later provide a chirally selective surface for biomolecules to crystallise on, or be a catalyst for chiral polymerisation to occur. Following Darwin's (1871) "warm little pond", an attractive scenario might be a tidal rock pool, 
where waves agitating pebbles provide the energetic input for grinding. Taking more account of recent work, a more likely place is a suboceanic hydrothermal vent where the rapid convection of hot water impels growing nucleii into the vent's rough walls as well as breaking particles off the walls and entraining them into the fluid flow, simultaneously grinding any growing crystals.

In "The BD Model with Dimer Interactions and an Amorphous Metastable Phase" we propose a detailed microscopic model of the nucleation and crystal growth of several species simultaneously. This has the form of a generalised Becker-Döring system of equations (1935). Due to the complexity of the model we immediately simplify it, making assumptions on the rate coefficients. Furthermore, to elucidate those processes which are responsible for homochiralisation, we remove some processes completely so as to obtain a simple system of ordinary differential equations which can be analysed theoretically.

The simplest model which might be expected to show homochiralisation is one which has small and large clusters of each handedness. Such a truncated model is considered in "The Truncation at Tetramers" wherein it is shown that such a model might lead to amplification of enantiomeric exess in the short time, but that in the long-time limit, only the racemic state can be approached. This model has the structure akin to that of Saito and Hyuga (2005) truncated at the tetramer level.

Hence, in "The Truncation at Hexamers" we consider a more complex model with a cut-off at larger sizes (one can think of small, medium, and large clusters of each handedness). Such a model has a similar structure to the hexamer truncation analysed by Saito and Hyuga (2005). We find that such a model does allow a final steady-state in which one chirality dominates the system and the other is present only in vanishingly small amounts.

However, as discussed earlier, there may be subtle effects whereby it is not just the number of crystals of each type that is important to the effect, but a combination of size and number of each handedness of crystal that is important to the evolution of the process. Hence, in "New Simplifications of the System" we introduce an alternative reduction of the system of governing equations. In this, instead of truncating and keeping only clusters of a small size, we postulate a form for the distribution which includes information on both the number and size of crystals, and use these two quantities to construct a system of five ordinary differential equations for the system's evolution.

We discuss the results in "Discussion" and "Conclusions" which conclude the paper. The Appendix A shows how, by removing the symmetry in the growth rates of the two handednesses, the model could be generalised to account for the competitive nucleation of different polymorphs growing from a common supply of monomer.

\section{The BD Model with Dimer Interactions and an Amorphous Metastable Phase}

\section{Preliminaries}

Smoluchowski (1916) proposed a model in which clusters of any sizes could combine pairwise to form larger clusters. Chemically this process is written $C_{r}+C_{s} \rightarrow C_{r+s}$ 
where $C_{r}$ represents a cluster of size $r$. Assuming this process is reversible and occurs with a forward rate given by $a_{r, s}$ and a reverse rate given by $b_{r, s}$, the law of mass action yields the kinetic equations

$$
\frac{\mathrm{d} c_{r}}{\mathrm{~d} t}=\frac{1}{2} \sum_{s=1}^{r-1}\left(a_{s, r-s} c_{s} c_{r-s}-b_{s, r-s} c_{r}\right)-\sum_{s=1}^{\infty}\left(a_{r, s} c_{r} c_{s}-b_{r, s} c_{r+s}\right) .
$$

These are known as the coagulation-fragmentation equations. There are simplifications in which only interactions between clusters of particular sizes are permitted to occur, for example when only cluster-monomer interactions can occur, the Becker-Döring equations (1935) are obtained. da Costa (1998) has formulated a system in which only clusters upto a certain size $(N)$ are permitted to coalesce with or fragment from other clusters. In the case of $N=2$, which is pertinent to the current study, only cluster-monomer and cluster-dimer interactions are allowed, for example

$$
C_{r}+C_{1} \rightleftharpoons C_{r+1}, \quad C_{r}+C_{2} \rightleftharpoons C_{r+2}
$$

This leads to a system of kinetic equations of the form

$$
\begin{aligned}
\frac{\mathrm{d} c_{r}}{\mathrm{~d} t} & =J_{r-1}-J_{r}+K_{r-2}-K_{r}, \quad(r \geq 3), \\
\frac{\mathrm{d} c_{2}}{\mathrm{~d} t} & =J_{1}-J_{2}-K_{2}-\sum_{r=1}^{\infty} K_{r}, \\
\frac{\mathrm{d} c_{1}}{\mathrm{~d} t} & =-J_{1}-K_{2}-\sum_{r=1}^{\infty} J_{r}, \\
J_{r} & =a_{r} c_{r} c_{1}-b_{r+1} c_{r+1}, \quad K_{r}=\alpha_{r} c_{r} c_{2}-\beta_{r+2} c_{r+2} .
\end{aligned}
$$

A simple example of such a system has been analysed previously by Bolton and Wattis (2002).

In the next subsection we generalise the model (Eq. 2.1) to include a variety of 'species' or 'morphologies' of cluster, representing left-handed, right-handed and achiral clusters. We simplify the model in stages to one in which only monomer and dimer interactions are described, and then one in which only dimer interactions occur.

\section{A Full Microscopic Model of Chiral Crystallisation}

We start by outlining all the possible cluster growth, fragmentation and transformation processes. We denote the two handed clusters by $X_{r}, Y_{r}$, where the subscript $r$ 
specifies the size of cluster. Achiral clusters are denoted by $C_{r}$, and we allow clusters to change their morphology spontaneously according to

$$
\begin{aligned}
& C_{r} \rightarrow X_{r} \text { rate }=\mu_{r}, \quad X_{r} \rightarrow C_{r} \text { rate }=\mu_{r} v_{r}, \\
& C_{r} \rightarrow Y_{r} \text { rate }=\mu_{r}, \quad Y_{r} \rightarrow C_{r} \text { rate }=\mu_{r} v_{r} .
\end{aligned}
$$

We allow clusters to grow by coalescing with clusters of similar handedness or an achiral cluster. In the case of the latter process, we assume that the cluster produced is chiral with the same chirality as the parent. Thus

$$
\begin{gathered}
X_{r}+X_{s} \rightarrow X_{r+s}, \quad \text { rate }=\xi_{r, s}, \\
X_{r}+C_{s} \rightarrow X_{r+s}, \quad \text { rate }=\alpha_{r, s}, \\
C_{r}+C_{s} \rightarrow C_{r+s}, \quad \text { rate }=\delta_{r, s}, \\
Y_{r}+C_{s} \rightarrow Y_{r+s}, \quad \text { rate }=\alpha_{r, s}, \\
Y_{r}+Y_{s} \rightarrow Y_{r+s}, \quad \text { rate }=\xi_{r, s} .
\end{gathered}
$$

We do not permit clusters of opposite to chirality to merge. Finally we describe fragmentation: all clusters may fragment, producing two smaller clusters each of the same chirality as the parent cluster

$$
\begin{gathered}
X_{r+s} \rightarrow X_{r}+X_{s} \text { rate }=\beta_{r, s}, \\
C_{r+s} \rightarrow C_{r}+C_{s} \quad \text { rate }=\epsilon_{r, s}, \\
Y_{r+s} \rightarrow Y_{r}+Y_{s} \quad \text { rate }=\beta_{r, s} .
\end{gathered}
$$

Setting up concentration variables for each size and each type of cluster by defining $c_{r}(t)=\left[C_{r}\right], x_{r}(t)=\left[X_{r}\right], y_{r}(t)=\left[Y_{r}\right]$ and applying the law of mass action, we obtain

$$
\begin{aligned}
\frac{\mathrm{d} c_{r}}{\mathrm{~d} t}= & -2 \mu_{r} c_{r}+\mu_{r} v_{r}\left(x_{r}+y_{r}\right)-\sum_{k=1}^{\infty} \alpha_{k, r} c_{r}\left(x_{k}+y_{k}\right) \\
& +\frac{1}{2} \sum_{k=1}^{r-1}\left(\delta_{k, r-k} c_{k} c_{r-k}-\epsilon_{k, r-k} c_{k} c_{r-k}\right)-\sum_{k=1}^{\infty}\left(\delta_{k, r} c_{k} c_{r}-\epsilon_{k, r} c_{r+k}\right), \\
\frac{\mathrm{d} x_{r}}{\mathrm{~d} t}= & \mu_{r} c_{r}-\mu_{r} v_{r} x_{r}+\sum_{k=1}^{r-1} \alpha_{k, r-k} c_{k} x_{r-k}-\frac{1}{2} \sum_{k=1}^{r-1}\left(\xi_{k, r-k} x_{k} x_{r-k}-\beta_{k, r-k} x_{r}\right) \\
& -\sum_{k=1}^{\infty}\left(\xi_{k, r} x_{k} x_{r}-\beta_{k, r} x_{r+k}\right), \\
\frac{\mathrm{d} y_{r}}{\mathrm{~d} t}= & \mu_{r} c_{r}-\mu_{r} v_{r} y_{r}+\sum_{k=1}^{r-1} \alpha_{k, r-k} c_{k} y_{r-k}-\frac{1}{2} \sum_{k=1}^{r-1}\left(\xi_{k, r-k} y_{k} y_{r-k}-\beta_{k, r-k} y_{r}\right) \\
& -\sum_{k=1}^{\infty}\left(\xi_{k, r} y_{k} y_{r}-\beta_{k, r} y_{r+k}\right) .
\end{aligned}
$$


The main problem with such a model is the vast number of parameters that have been introduced $\left(\alpha_{r, k}, \xi_{r, k}, \beta_{r, k}, \mu_{r}, v_{r}, \delta_{r, k}, \epsilon_{r, k}\right.$, for all $\left.k, r\right)$.

Hence we make several simplifications:

(i) we assume that the dominant coagulation and fragmentation processes are between large and very small clusters (rather than large clusters and other large clusters). Specifically, we assume that only coalescences involving $C_{1}$ and $C_{2}$ need to be retained in the model, and fragmentation always yields either a monomer or a dimer fragment. This assumption means that the system can be reduced to a generalised Becker-Döring equation closer to the form of Eqs. 2.3-2.6 rather than Eq. 2.1;

(ii) we also assume that the achiral clusters are unstable at larger size, so that their presence is only relevant at small sizes. Typically at small sizes, clusters are amorphous and do not take on the properties of the bulk phase, hence at small sizes clusters can be considered achiral. We assume that there is a regime of cluster sizes where there is a transition to chiral structures, and where clusters can take on the bulk structure (which is chiral) as well as exist in amorphous form. At even larger sizes, we assume that only the chiral forms exist, and no achiral structure can be adopted;

(iii) furthermore, we assume that all rates are independent of cluster size, specifically,

$$
\begin{aligned}
& \alpha_{k, 1}=a, \quad \alpha_{k, 2}=\alpha, \quad \alpha_{k, r}=0, \quad(r \geq 2) \\
& \mu_{2}=\mu, \quad \mu_{r}=0, \quad(r \geq 3), \\
& v_{2}=v, \quad \quad v_{r}=0, \quad(r \geq 3), \\
& \delta_{1,1}=\delta, \quad \delta_{k, r}=0, \quad \text { (otherwise) } \\
& \epsilon_{1,1}=\epsilon, \quad \epsilon_{k, r}=0, \quad \text { (otherwise) } \\
& \xi_{k, 2}=\xi_{2, k}=\xi, \quad \xi_{k, r}=0, \quad \text { (otherwise) } \\
& \beta_{k, 1}=\beta_{1, k}=b, \quad \beta_{k, 2}=\beta_{2, k}=\beta, \quad \beta_{k, r}=0, \quad \text { (otherwise), }
\end{aligned}
$$

Ultimately we will set $a=b=0=\delta=\epsilon$ so that we have only five parameters to consider $(\alpha, \xi, \beta, \mu, v)$.

This scheme is illustrated in Fig. 1. However, before writing down a further system of equations, we make one further simplification. We take the transition region described in (ii), above, to be just the dimers. Thus the only types of achiral cluster are the monomer and the dimer $\left(c_{1}, c_{2}\right)$; dimers exist in achiral, right- and 
Fig. 1 Reaction scheme involving monomer and dimer aggregation and fragmentation of achiral clusters and those of both handednesses (right and left). The aggregation of achiral and chiral clusters is not shown (rates $\alpha, \xi)$

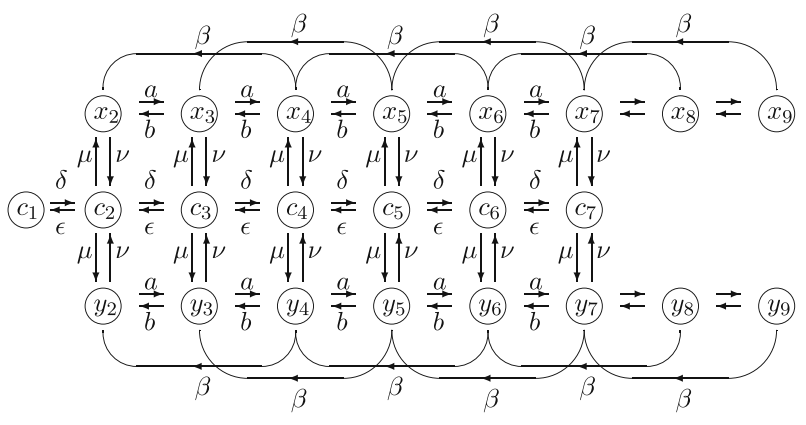

left-handed forms $\left(c_{2}, x_{2}, y_{2}\right)$; at larger sizes only left- and right-handed clusters exist $\left(x_{r}, y_{r}, r \geq 2\right)$.

The kinetic equations can be reduced to

$$
\begin{aligned}
& \frac{\mathrm{d} c_{1}}{\mathrm{~d} t}=2 \varepsilon c_{2}-2 \delta c_{1}^{2}-\sum_{r=2}^{\infty}\left(a c_{1} x_{r}+a c_{1} y_{r}-b x_{r+1}-b y_{r+1}\right), \\
& \frac{\mathrm{d} c_{2}}{\mathrm{~d} t}=\delta c_{1}^{2}-\varepsilon c_{2}-2 \mu c_{2}+\mu \nu\left(x_{2}+y_{2}\right)-\sum_{r=2}^{\infty} \alpha c_{2}\left(x_{r}+y_{r}\right), \\
& \frac{\mathrm{d} x_{r}}{\mathrm{~d} t}=a c_{1} x_{r-1}-b x_{r}-a c_{1} x_{r}+b x_{r+1}+\alpha c_{2} x_{r-2}-\alpha c_{2} x_{r} \\
& -\beta x_{r}+\beta x_{r+2}+\xi x_{2} x_{r-2}-\xi x_{2} x_{r}, \quad(r \geq 4), \\
& \frac{\mathrm{d} x_{3}}{\mathrm{~d} t}=a c_{1} x_{2}-b x_{3}-a c_{1} x_{3}+b x_{4}-\alpha c_{2} x_{3}-\xi x_{2} x_{3}+\beta x_{5}, \\
& \frac{\mathrm{d} x_{2}}{\mathrm{~d} t}=\mu c_{2}-\mu \nu x_{2}+b x_{3}-a c_{1} x_{2}-\alpha x_{2} c_{2}+\beta x_{4} \\
& +\sum_{r=2}^{\infty} \beta x_{r+2}-\sum_{r=2}^{\infty} \xi x_{2} x_{r}-\xi x_{2}^{2} \\
& \frac{\mathrm{d} y_{r}}{\mathrm{~d} t}=a c_{1} y_{r-1}-b y_{r}-a c_{1} y_{r}+b y_{r+1}+\alpha c_{2} y_{r-2}-\alpha c_{2} y_{r} \\
& -\beta y_{r}+\beta y_{r+2}+\xi y_{2} y_{r-2}-\xi y_{2} y_{r}, \quad(r \geq 4), \\
& \frac{\mathrm{d} y_{3}}{\mathrm{~d} t}=a c_{1} y_{2}-b y_{3}-a c_{1} y_{3}+b y_{4}-\alpha c_{2} y_{3}-\xi y_{2} y_{3}+\beta y_{5} \text {, } \\
& \frac{\mathrm{d} y_{2}}{\mathrm{~d} t}=\mu c_{2}-\mu \nu y_{2}+b y_{3}-a c_{1} y_{2}-\alpha y_{2} c_{2}+\beta y_{4} \\
& +\sum_{r=2}^{\infty} \beta y_{r+2}-\sum_{r=2}^{\infty} \xi y_{2} y_{r}-\xi y_{2}^{2} \text {. }
\end{aligned}
$$


Summary and Simulations of the Macroscopic Model

The advantage of the above simplifications is that certain sums appear repeatedly; by defining new quantities as these sums, the system can be written in a simpler fashion. We define $N_{x}=\sum_{r=2}^{\infty} x_{r}, N_{y}=\sum_{r=2}^{\infty} y_{r}$, then

$$
\begin{aligned}
\frac{\mathrm{d} c_{1}}{\mathrm{~d} t}= & 2 \varepsilon c_{2}-2 \delta c_{1}^{2}-a c_{1}\left(N_{x}+N_{y}\right)+b\left(N_{x}-x_{2}+N_{y}-y_{2}\right), \\
\frac{\mathrm{d} c_{2}}{\mathrm{~d} t}= & \delta c_{1}^{2}-\varepsilon c_{2}-2 \mu c_{2}+\mu \nu\left(x_{2}+y_{2}\right)-\alpha c_{2}\left(N_{x}+N_{y}\right), \\
\frac{\mathrm{d} N_{x}}{\mathrm{~d} t}= & \mu c_{2}-\mu \nu x_{2}+\beta\left(N_{x}-x_{3}-x_{2}\right)-\xi x_{2} N_{x}, \\
\frac{\mathrm{d} x_{2}}{\mathrm{~d} t}= & \mu c_{2}-\mu \nu x_{2}+b x_{3}-a c_{1} x_{2}-\alpha x_{2} c_{2}+\beta\left(x_{4}+N_{x}-x_{2}-x_{3}\right) \\
& -\xi x_{2}^{2}-\xi x_{2} N_{x}, \\
\frac{\mathrm{d} N_{y}}{\mathrm{~d} t}= & \mu c_{2}-\mu v y_{2}+\beta\left(N_{y}-y_{3}-y_{2}\right)-\xi y_{2} N_{y}, \\
\frac{\mathrm{d} y_{2}}{\mathrm{~d} t}= & \mu c_{2}-\mu v y_{2}+b y_{3}-a c_{1} y_{2}-\alpha y_{2} c_{2}+\beta\left(y_{4}+N_{y}-y_{2}-y_{3}\right) \\
& -\xi y_{2}^{2}-\xi y_{2} N_{y} .
\end{aligned}
$$

However, such a system of equations is not 'closed'. The equations contain $x_{3}, y_{3}, x_{4}, y_{4}$, and yet we have no expressions for these; reintroducing equations for $x_{3}, y_{3}$ would introduce $x_{5}, y_{5}$ and so an infinite regression would be entered into.

Hence we need to find some suitable alternative expressions for $x_{3}, y_{3}, x_{4}, y_{4}$; or an alternative way of reducing the system to just a few ordinary differential equations that can easily be analysed. Such systems are considered in "The Truncation at Tetramers", "The Truncation at Hexamers" and "New Simplifications of the System. Before that, however, we illustrate the behaviour of the system by briefly presenting the results of some numerical simulations. In Figs. 2 and 3 we show the results of a simulation of Eqs. 2.28-2.33. The former shows the evolution of the concentrations $c_{1}$ which rises then decays, $c_{2}$ which decays since the parameters have been chosen to reflect a cluster-dominated system. Also plotted are the numbers of clusters $N_{x}, N_{y}$ and the mass of material in clusters $\varrho_{x}, \varrho_{y}$ defined by

$$
\varrho_{x}=\sum_{j=2}^{K} j x_{j}, \quad \varrho_{y}=\sum_{j=2}^{K} j y_{j} .
$$

Note that under this definition $\varrho_{x}+\varrho_{y}+c_{1}+2 c_{2}$ is conserved, and this is plotted as rho. Both the total number of clusters, $N_{x}+N_{y}$, and total mass of material in handed clusters $\varrho_{x}+\varrho_{y}$ appear to equilibrate by $t=10^{2}$, however, at a much later time $\left(t \sim 10^{4}-10^{5}\right)$ a symmetry-breaking bifurcation occurs, and the system changes from almost racemic (that is, symmetric) to asymmetric. This is more clearly seen in Fig. 3, where we plot the cluster size distribution at three time points. At $t=0$ there are only dimers present (dashed line), and we impose a small difference in the 
Fig. 2 Plot of the concentrations $c_{1}, c_{2}, N_{x}, N_{y}$, $N=N_{x}+N_{y}, \varrho_{x}, \varrho_{y}, \varrho_{x}+\varrho_{y}$ and $\varrho_{x}+\varrho_{y}+2 c_{2}+c 1$ against time, $t$ on a logarithmic timescale. Since model equations are in nondimensional form, the time units are arbitrary. Parameter values $\mu=1.0, v=0.5, \delta=1$, $\varepsilon=5, a=4, b=0.02, \alpha=10$, $\xi=10, \beta=0.03$, with initial conditions $c_{2}=0.49$, $x_{4}(0)=0.004, y_{4}(0)=0.006$, and all other concentrations zero

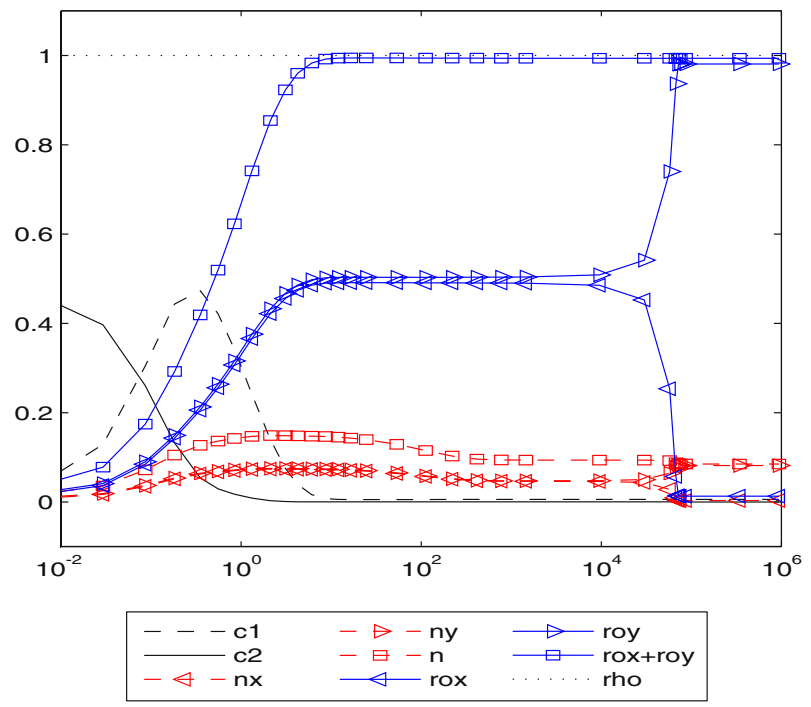

concentrations of $x_{2}$ and $y_{2}$. At a later time, $t=112$ (dotted line), there is almost no difference between the $X$-and $Y$-distributions, however by the end of the simulation $\left(t \sim 10^{6}\right.$, solid line) one distribution clearly completely dominates the other.

\section{Simplified Macroscopic Model}

To obtain the simplest model which involves three polymorphs corresponding to right-handed and left-handed chiral clusters and achiral clusters, we now aim to simplify the processes of cluster aggregation and fragmentation in Eqs. 2.28-2.33. Our aim is to retain the symmetry-breaking phenomenon but eliminate physical processes which are not necessary for it to occur.

Fig. 3 Plot of the cluster size distribution at $t=0$ (dashed line $), t=112$ (dotted line $)$ and $t=9.4 \times 10^{5}$. Parameters and initial conditions as in Fig. 2

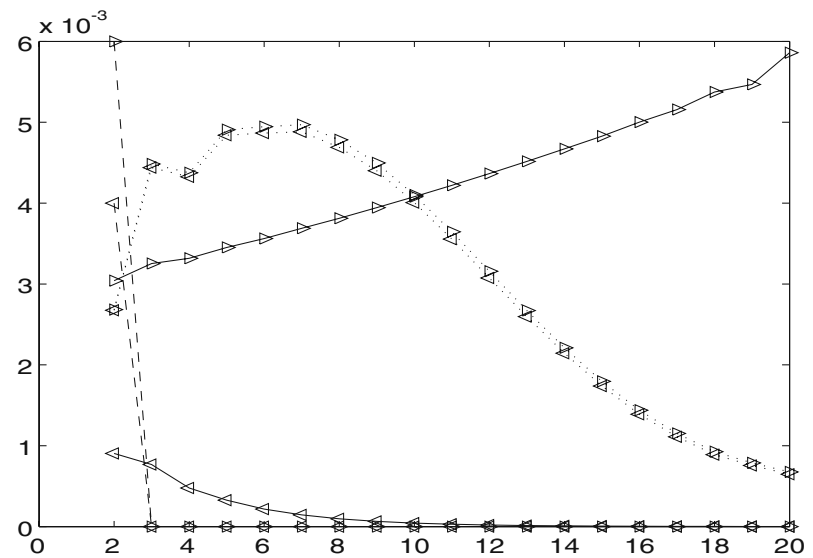


Our first simplification is to remove all clusters of odd size from the model, and just consider dimers, tetramers, hexamers, etc. This corresponds to putting $a=0$, $b=0$ which removes $x_{3}$ and $y_{3}$ from the system. Furthermore, we put $\varepsilon=0$ and make $\delta$ large, so that the achiral monomer is rapidly and irreversibly converted to achiral dimer. Since the monomers do not then influence the evolution of any of the other variables, we further simplify the system by ignoring $c_{1}$ (or, more simply, just impose initial data in which $\left.c_{1}(0)=0\right)$. Thus we are left with

$$
\begin{aligned}
\frac{\mathrm{d} c_{2}}{\mathrm{~d} t} & =-2 \mu c_{2}+\mu \nu\left(x_{2}+y_{2}\right)-\alpha c_{2}\left(N_{x}+N_{y}\right), \\
\frac{\mathrm{d} N_{x}}{\mathrm{~d} t} & =\mu c_{2}-\mu \nu x_{2}+\beta\left(N_{x}-x_{2}\right)-\xi x_{2} N_{x}, \\
\frac{\mathrm{d} x_{2}}{\mathrm{~d} t} & =\mu c_{2}-\mu \nu x_{2}-\alpha x_{2} c_{2}+\beta\left(N_{x}-x_{2}+x_{4}\right)-\xi x_{2}^{2}-\xi x_{2} N_{x}, \\
\frac{\mathrm{d} N_{y}}{\mathrm{~d} t} & =\mu c_{2}-\mu \nu y_{2}+\beta\left(N_{y}-y_{2}\right)-\xi y_{2} N_{y}, \\
\frac{\mathrm{d} y_{2}}{\mathrm{~d} t} & =\mu c_{2}-\mu \nu y_{2}-\alpha y_{2} c_{2}+\beta\left(N_{y}-y_{2}+y_{4}\right)-\xi y_{2}^{2}-\xi y_{2} N_{y} .
\end{aligned}
$$

Since we have removed four parameters from the model, and halved the number of dependent variables, we show a couple of numerical simulations just to show that the system above does still exhibit symmetry-breaking behaviour.

Figure 4 appears similar to Fig. 2, suggesting that removing the monomer interactions has changed the underlying dynamics little. We still observe the characteristic

Fig. 4 Plot of the concentrations $c_{1}, c_{2}, N_{x}, N_{y}$, $N=N_{x}+N_{y}, \varrho_{x}, \varrho_{y}, \varrho_{x}+\varrho_{y}$ and $\varrho_{x}+\varrho_{y}+2 c_{2}+c_{1}$ against time, $t$ on a logarithmic timescale. Since model equations are in nondimensional form, the time units are arbitrary. Parameter values $\mu=1, v=0.5, \alpha=10$, $\xi=10, \beta=0.03$, with initial conditions $c_{2}=0.49$,

$x_{4}(0)=0.004, y_{4}(0)=0.006$, all other concentrations zero

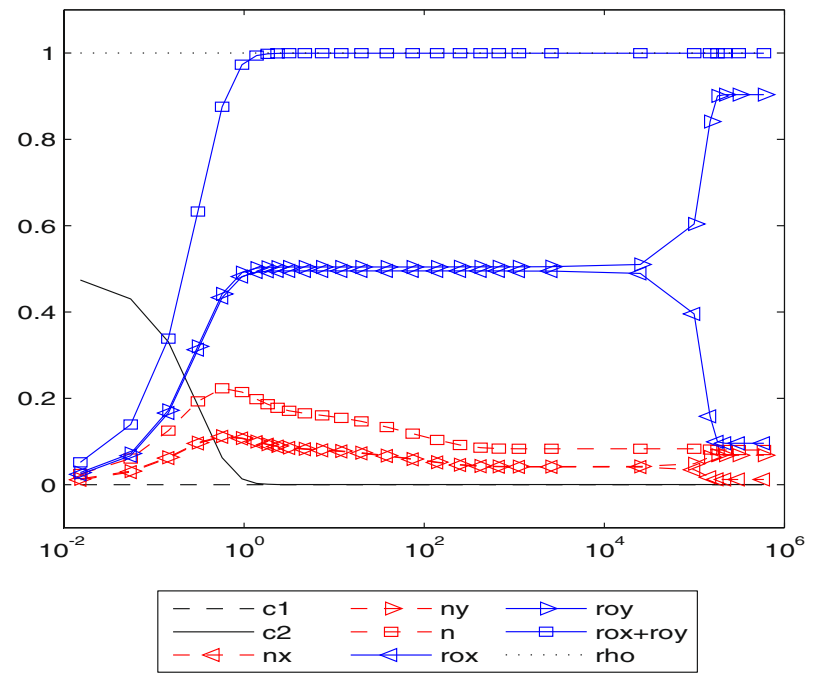


Fig. 5 Plot of the cluster size distribution at $t=0$ (dashed line $), t=250$ (dotted line) and $t=6 \times 10^{5}$. Parameters and initial conditions as in Fig. 4

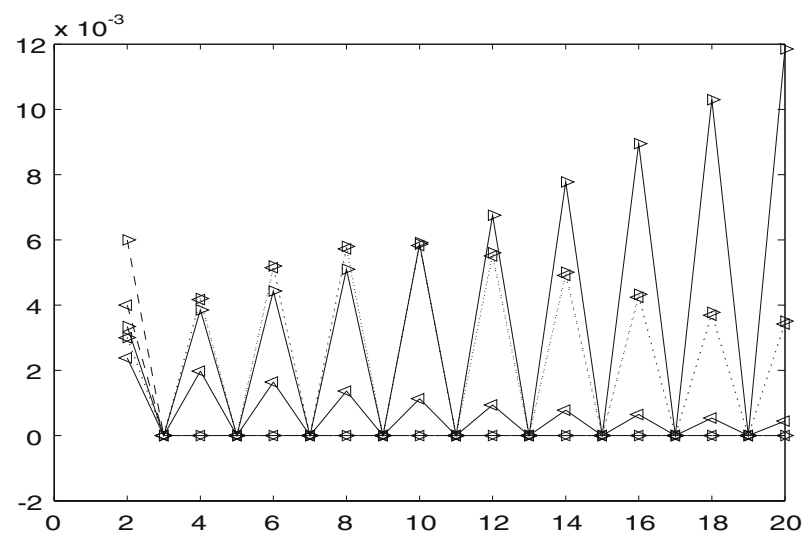

equilibration of cluster numbers and cluster masses as $c_{2}$ decays, and then a period of quiesence $\left(t \sim 10\right.$ to $\left.10^{4}\right)$ before a later symmetry-breaking event, around $t \sim 10^{5}$. At first sight, the distribution of $X$ - and $Y$-clusters displayed in Fig. 5 is quite different to Fig. 3; this is due to the absence of monomers from the system, meaning that only even-sized clusters can now be formed. If one only looks at the even-sized clusters in Fig. 5, we once again see only a slight difference at $t=0$ (dashed line), almost no difference at $t \approx 250$ (dotted line) but a significant difference at $t=6 \times 10^{5}$ (solid line). We include one further graph here, Fig. 6 similar to Fig. 4 but on a linear rather than a logarithmic timescale. This should be compared with figures such as Figs. 3 and 4 of Viedma (2005) and Fig. 1 of Noorduin et al. (2008).

Fig. 6 Plot of the concentrations $c_{1}, c_{2}, N_{x}, N_{y}$, $N=N_{x}+N_{y}, \varrho_{x}, \varrho_{y}, \varrho_{x}+\varrho_{y}$ and $\varrho_{x}+\varrho_{y}+2 c_{2}+c_{1}$ against time, $t$ on a logarithmic timescale. Parameters and initial conditions as in Fig. 4

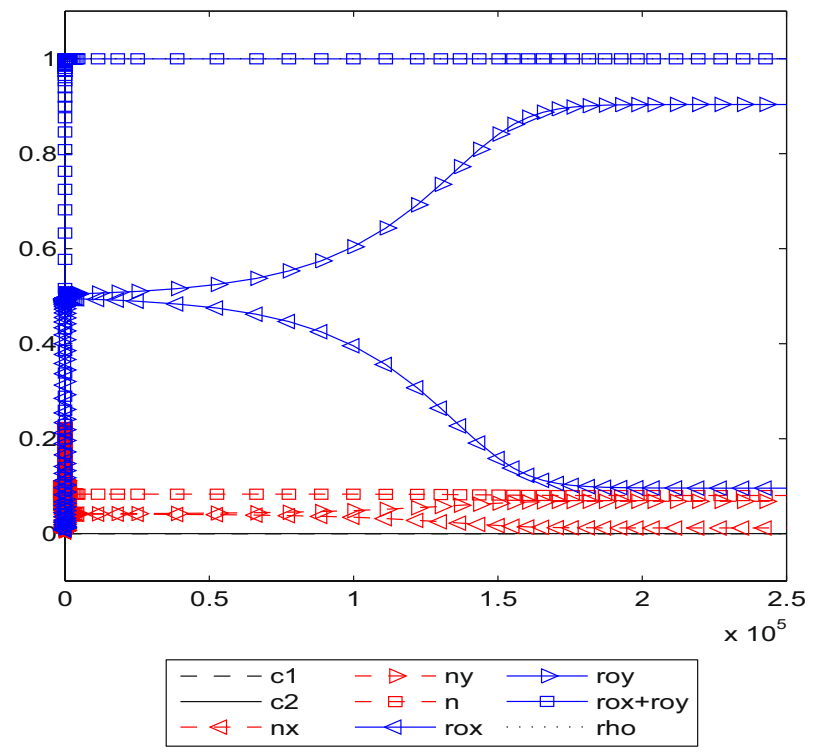




\section{The Truncation at Tetramers}

The simplest possible reaction scheme of the form Eqs. 2.20-2.27 which we might expect to exhibit symmetry-breaking to homochirality is the system truncated at tetramers (Fig. 7), namely

$$
\begin{aligned}
& \frac{\mathrm{d} c_{2}}{\mathrm{~d} t}=-2 \mu c_{2}+\mu \nu\left(x_{2}+y_{2}\right)-\alpha c_{2}\left(x_{2}+y_{2}\right), \\
& \frac{\mathrm{d} x_{2}}{\mathrm{~d} t}=\mu c_{2}-\mu \nu x_{2}-\alpha c_{2} x_{2}-2 \xi x_{2}^{2}+2 \beta x_{4}, \\
& \frac{\mathrm{d} y_{2}}{\mathrm{~d} t}=\mu c_{2}-\mu \nu y_{2}-\alpha c_{2} y_{2}-2 \xi y_{2}^{2}+2 \beta y_{4}, \\
& \frac{\mathrm{d} x_{4}}{\mathrm{~d} t}=\alpha x_{2} c_{2}+\xi x_{2}^{2}-\beta x_{4} \\
& \frac{\mathrm{d} y_{4}}{\mathrm{~d} t}=\alpha y_{2} c_{2}+\xi y_{2}^{2}-\beta y_{4} .
\end{aligned}
$$

We investigate the symmetry-breaking by transforming the variables $x_{2}, x_{4}, y_{2}, y_{4}$ according to

$$
\begin{aligned}
x_{2} & =\frac{1}{2} z(1+\theta), & y_{2} & =\frac{1}{2} z(1-\theta), \\
x_{4} & =\frac{1}{2} w(1+\phi), & y_{4} & =\frac{1}{2} w(1-\phi),
\end{aligned}
$$

Fig. 7 Simplest possible reaction scheme which might exhibit chiral symmetry-breaking

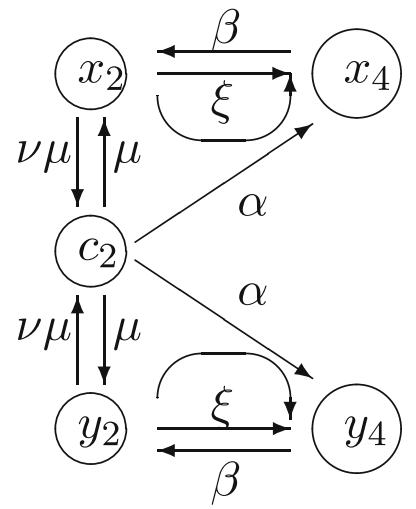


where $z=x_{2}+y_{2}$ is the total concentration of chiral dimers, $w=x_{4}+y_{4}$ is the total tetramer concentration, $\theta=\left(x_{2}-y_{2}\right) / z$ is the relative chirality of the dimers, $\phi=$ $\left(x_{4}-y_{4}\right) / w$ is the relative chirality of tetramers. Hence

$$
\begin{aligned}
& \frac{\mathrm{d} c_{2}}{\mathrm{~d} t}=-2 \mu c_{2}+\mu \nu z-\alpha c_{2} z, \\
& \frac{\mathrm{d} z}{\mathrm{~d} t}=2 \mu c_{2}-\mu \nu z-\alpha c_{2} z-\xi z^{2}\left(1+\theta^{2}\right)+2 \beta w, \\
& \frac{\mathrm{d} w}{\mathrm{~d} t}=\alpha z c_{2}+\frac{1}{2} \xi z^{2}\left(1+\theta^{2}\right)-\beta w, \\
& \frac{\mathrm{d} \theta}{\mathrm{d} t}=-\theta\left(\frac{2 \mu c}{z}+\frac{2 \beta w}{z}+\xi z\left(1-\theta^{2}\right)\right)+\frac{2 \beta w \phi}{z}, \\
& \frac{\mathrm{d} \phi}{\mathrm{d} t}=\theta \frac{z}{w}(\alpha c+\xi z)-\left(\alpha c+\frac{1}{2} \xi z\left(1+\theta^{2}\right)\right) \frac{z}{w} \phi .
\end{aligned}
$$

The stability of the evolving symmetric-state $(\theta=\phi=0)$ is given by the eigenvalues (q) of the matrix

$$
\left(\begin{array}{cc}
-\left(\frac{2 \mu c}{z}+\frac{2 \beta w}{z}+\xi z\right) & \frac{2 \beta w}{z} \\
(\alpha c+\xi z) \frac{z}{w} & -\left(\alpha c+\frac{1}{2} \xi z\right) \frac{z}{w}
\end{array}\right)
$$

which are given by

$$
\begin{gathered}
q^{2}+q\left(\frac{\alpha c z}{w}+\frac{\xi z^{2}}{w}+\frac{2 \mu c}{z}+\xi z+\frac{2 \beta w}{z}\right)+ \\
\frac{1}{w}\left(2 \mu \alpha c^{2}+\mu c \xi z+\alpha c \xi z^{2}+\frac{1}{2} \xi^{2} z^{3}-\beta \xi z w\right)=0 .
\end{gathered}
$$

Hence there is an instability if

$$
\beta \xi z w>2 \mu \alpha c^{2}+\mu c \xi z+\alpha c \xi z^{2}+\frac{1}{2} \xi^{2} z^{3}
$$

using the steady-state result that $2 \beta w=z(2 \alpha c+\xi z)$ and factorising $(2 \alpha c+\xi z)$ out of the result, reduces the instability Eq. 3.15 to the contradictory $\xi z^{2}>\xi z^{2}+2 \mu c$. Hence the racemic steady-state of the system is stable for all choices of parameter values and is approached from all initial conditions. However, initial perturbations, may be amplified due to the presence of nonlinear terms. 
Fig. 8 The concentrations $c_{2}$, $z$ and $w$ Eqs. 3.6-3.7 plotted against time, for the tetramer-truncated system with the two sets of initial data (Eq. 3.16). Since model equations are in nondimensional form, the time units are arbitrary. The parameter values are $\mu=1$, $v=0.5, \alpha=\xi=10, \beta=0.1$

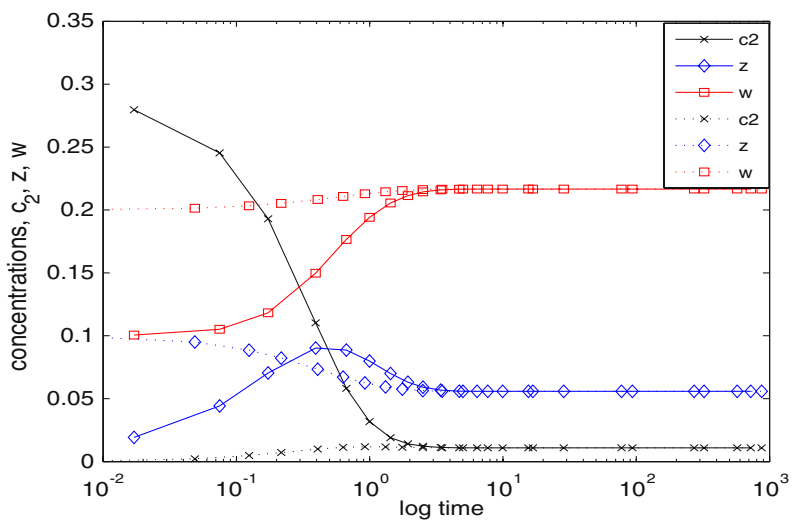

Evolution from two sets of initial conditions of the system Eqs. 3.1-3.5 are shown in each of Figs. 8 and 9. The continuous and dotted lines correspond to the initial data

$$
\begin{gathered}
c_{2}(0)=0.29, \quad x_{2}(0)=0.0051, \quad y_{2}(0)=0.0049 \\
x_{4}(0)=0.051, \quad y_{4}(0)=0.049 ; \quad \text { and } \\
c_{2}(0)=0, \quad x_{2}(0)=0.051 \quad y_{2}(0)=0.049 \\
x_{4}(0)=0.1, \quad y_{4}(0)=0.1
\end{gathered}
$$

respectively. In the former case, the system starts with considerable amount of amorphous dimer, which is converted into clusters, and initially there is a slight chiral imbalance in favour of $x_{2}$ and $x_{4}$ over $y_{2}$ and $y_{4}$. Over time this imbalance reduces (see Fig. 9); although there is a region around $t=1$ where $\theta$ increases, both $\theta$ and $\phi$ eventually approach the zero steady-state.

For both sets of initial conditions we note that the chiralities evolve over a significantly longer timescale than the concentrations, the latter having reached steady-state before $t=10$ and the former still evolving when $t=\mathcal{O}\left(10^{2}\right)$. In the second set of initial data, there is no $c_{2}$ present initially and there are exactly equal

Fig. 9 The chiralities $\theta, \phi$ Eqs. 3.6-3.7 plotted against time, for the tetramer-truncated system with the two sets of initial data (Eq. 3.16). Since model equations are in nondimensional form, the time units are arbitrary. The parameter values are the same as in Fig. 8

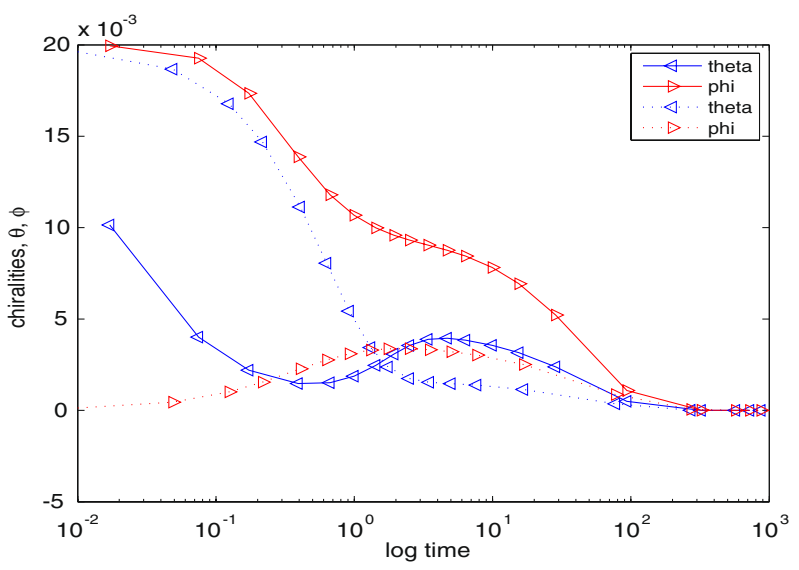


numbers of the two chiral forms of the larger cluster, but a slight exess of $x_{2}$ over $y_{2}$. In time an imbalance in larger clusters is produced, but over larger timescales, both $\theta$ and $\phi$ again approach the zero steady-state.

Hence, we observe that the truncated system Eqs. 3.1-3.5 does not yield a chirally asymmetric steady-state. Even though in the early stages of the reaction chiral perturbations may be amplified, at the end of the reaction there is a slower timescale over which the system returns to a racemic state. In the next section we consider a system truncated at hexamers to investigate whether that system allows symmetrybreaking of the steady-state.

\section{The Truncation at Hexamers}

The above analysis has shown that the truncation of the model Eqs. 2.20-2.27 to Eqs. 3.1-3.5 results in a model which always ultimately approaches the symmetric (racemic) steady-state. In this section, we show that a more complex model, the truncation at hexamers retains enough complexity to demonstrate the symmetrybreaking bifurcation which occurs in the full system. In this case the governing equations are

$$
\begin{aligned}
& \frac{\mathrm{d} c_{2}}{\mathrm{~d} t}=-2 \mu c_{2}+\mu \nu\left(x_{2}+y_{2}\right)-\alpha c_{2}\left(x_{2}+y_{2}\right)-\alpha c_{2}\left(x_{4}+y_{4}\right), \\
& \frac{\mathrm{d} x_{2}}{\mathrm{~d} t}=\mu c_{2}-\mu \nu x_{2}-\alpha c_{2} x_{2}-2 \xi x_{2}^{2}-\xi x_{2} x_{4}+2 \beta x_{4}+\beta x_{6}, \\
& \frac{\mathrm{d} x_{4}}{\mathrm{~d} t}=\alpha x_{2} c_{2}+\xi x_{2}^{2}-\beta x_{4}-\alpha c_{2} x_{4}-\xi x_{2} x_{4}+\beta x_{6}, \\
& \frac{\mathrm{d} x_{6}}{\mathrm{~d} t}=\alpha x_{4} c_{2}+\xi x_{2} x_{4}-\beta x_{6}, \\
& \frac{\mathrm{d} y_{2}}{\mathrm{~d} t}=\mu c_{2}-\mu \nu y_{2}-\alpha c_{2} y_{2}-2 \xi y_{2}^{2}-\xi y_{2} y_{4}+2 \beta y_{4}+\beta y_{6}, \\
& \frac{\mathrm{d} y_{4}}{\mathrm{~d} t}=\alpha y_{2} c_{2}+\xi y_{2}^{2}-\beta y_{4}-\alpha c_{2} y_{4}-\xi y_{2} y_{4}+\beta y_{6}, \\
& \frac{\mathrm{d} y_{6}}{\mathrm{~d} t}=\alpha y_{4} c_{2}+\xi y_{2} y_{4}-\beta y_{6} .
\end{aligned}
$$

To analyse the symmetry-breaking in the system we transform the dependent coordinates from $x_{2}, x_{4}, x_{6}, y_{2}, y_{4}, y_{6}$ to total concentrations $z, w, u$ and relative chiralities $\theta, \phi, \psi$ according to

$$
\begin{aligned}
x_{2} & =\frac{1}{2} z(1+\theta), & x_{4} & =\frac{1}{2} w(1+\phi), \\
y_{2} & =\frac{1}{2} z(1-\theta), & x_{6} & =\frac{1}{2} u(1+\psi), \\
y_{4} & =\frac{1}{2} w(1-\phi), & y_{6} & =\frac{1}{2} u(1-\psi) .
\end{aligned}
$$


We now separate the governing equations for the total concentrations of dimers $(c, z)$, tetramers $(w)$ and hexamers $(u)$

$$
\begin{aligned}
& \frac{\mathrm{d} c}{\mathrm{~d} t}=-2 \mu c+\mu \nu z-\alpha c z-\alpha c w, \\
& \frac{\mathrm{d} z}{\mathrm{~d} t}=2 \mu c-\mu \nu z-\alpha c z-\xi z^{2}\left(1+\theta^{2}\right)-\frac{1}{2} z w(1+\theta \phi)+\beta u+2 \beta w, \\
& \frac{\mathrm{d} w}{\mathrm{~d} t}=\alpha c z+\frac{1}{2} \xi z^{2}\left(1+\theta^{2}\right)-\beta w+\beta u-\alpha c w-\frac{1}{2} \xi z w(1+\theta \phi), \\
& \frac{\mathrm{d} u}{\mathrm{~d} t}=\alpha c w+\frac{1}{2} \xi z w(1+\theta \phi)-\beta u,
\end{aligned}
$$

from those for the chiralities

$$
\begin{aligned}
\frac{\mathrm{d} \psi}{\mathrm{d} t}= & \frac{\alpha c w}{u}(\phi-\psi)+\frac{\xi z w}{2 u}(\theta+\phi-\psi-\psi \phi \theta) \\
\frac{\mathrm{d} \phi}{\mathrm{d} t}= & \frac{\alpha c z}{w}(\theta-\phi)+\frac{\xi z^{2}}{2 w}\left(2 \theta-\phi-\phi \theta^{2}\right)+\frac{\beta u}{w}(\psi-\phi)-\frac{1}{2} \xi z \theta\left(1-\phi^{2}\right), \\
\frac{\mathrm{d} \theta}{\mathrm{d} t}= & -\frac{2 \mu c \theta}{z}-\xi z \theta\left(1-\theta^{2}\right)-\frac{1}{2} \xi w \phi\left(1-\theta^{2}\right)+\frac{\beta u \psi}{z}-\frac{\beta u \theta}{z} \\
& +\frac{2 \beta w \phi}{z}-\frac{2 \beta w \theta}{z} .
\end{aligned}
$$

In applications, we expect $v<1$, so that the small amorphous clusters (dimers) prefer to adopt one of their chiral states rather than the achiral structure. In addition, we note that the grinding process observed in experiments is much longer than the crystallisation process, and that there are many larger, macroscopic crystals hence we consider two limits in which $\beta \ll \alpha \xi$. We will consider the case of small $\beta$ with all other parameters being $\mathcal{O}(1)$ and then the case where $\alpha \sim \xi \gg 1$ and all other parameters are $\mathcal{O}(1)$.

\section{Symmetric Steady-State for the Concentrations}

Firstly, let us solve for the symmetric steady-state. In this case we assume $\theta=0=$ $\phi=\psi$, simplifying Eqs. 4.9-4.12. One of these is a redundant equation, hence we have the solution

$$
\begin{gathered}
w=\frac{z}{\beta}\left(\alpha c+\frac{1}{2} \xi z\right), \quad u=\frac{z}{\beta^{2}}\left(\alpha c+\frac{1}{2} \xi z\right)^{2}, \\
c=\frac{1}{\alpha}\left(\sqrt{\left(\frac{\beta}{2}+\frac{\beta \mu}{\alpha z}+\frac{\xi z}{4}\right)^{2}+\beta \mu \nu}-\frac{\beta}{2}-\frac{\beta \mu}{\alpha z}-\frac{\xi z}{4}\right),
\end{gathered}
$$


with $z$ being determined by conservation of total mass in the system

$$
2 c+2 z+4 w+6 u=\varrho .
$$

In the case of small grinding, $(\beta \ll 1)$, with $\varrho$ and all other parameters being $\mathcal{O}(1)$, we find

$$
\begin{aligned}
z & =\left(\frac{2 \varrho \beta^{2}}{3(\alpha \nu+\xi)^{2}}\right)^{1 / 3}, & c & =v\left(\frac{\varrho \beta^{2}}{12(\alpha \nu+\xi)^{2}}\right)^{1 / 3}, \\
w & =\left(\frac{\varrho^{2} \beta}{18(\alpha \nu+\xi)}\right)^{1 / 3}, & u & =\frac{\varrho}{6} .
\end{aligned}
$$

In this case most of the mass is in hexamers with a little in tetramers and very little in dimers.

In the asymptotic limit of $\alpha \sim \xi \gg 1$ and all other parameters $\mathcal{O}(1)$, we find

$$
c=\frac{\mu \nu}{\alpha}\left(\frac{12 \beta}{\varrho \xi}\right)^{1 / 3}, \quad z=\left(\frac{2 \beta^{2} \varrho}{3 \xi^{2}}\right)^{1 / 3}, \quad w=\left(\frac{\beta \varrho^{2}}{18 \xi}\right)^{1 / 3}, \quad u=\frac{\varrho}{6}
$$

This differs significantly from the other asymptotic scaling as, not only are $c$ and $z$ both small, they are now different orders of magnitude, with $c \ll z$. We next analyse the stability of these symmetric states.

\section{Stability of Symmetric State}

In deriving the above solutions (Eqs. 4.16-4.17), we have assumed chiral symmetry, that is, $\theta=0=\psi=\phi$. We now turn to analyse the validity of this assumption. Linearising the system of Eqs. 4.13-4.15 which govern the chiralities, we determine whether the symmetric solution is stable from

$$
\frac{\mathrm{d}}{\mathrm{d} t}\left(\begin{array}{l}
\psi \\
\phi \\
\theta
\end{array}\right)=\left(\begin{array}{ccc}
-\frac{\alpha c w}{u}-\frac{\xi z w}{2 u} & \frac{\alpha c w}{u}+\frac{\xi z w}{2 u} & \frac{\xi z w}{2 u} \\
\frac{\beta u}{w} & -\frac{\alpha c z}{w}-\frac{\xi z^{2}}{2 w}-\frac{\beta u}{w} & \frac{\alpha c z}{w}+\frac{\xi z^{2}}{w}-\frac{1}{2} \xi z \\
\frac{\beta u}{z} & \frac{2 \beta w}{z}-\frac{\xi w}{2} & -\frac{2 \mu c}{z}-\xi z-\frac{\beta u}{z}-\frac{2 \beta w}{z}
\end{array}\right)\left(\begin{array}{c}
\psi \\
\phi \\
\theta
\end{array}\right) .
$$

For later calculations it is useful to know the determinant of this matrix. Using the steady-state solutions (Eq. 4.16), the determinant simplifies to

$$
D=\frac{3 c}{4 \beta \rho}(2 \alpha c+\xi z)^{2}\left(\alpha \xi z^{2}-4 \beta \mu\right)
$$


For general parameter values, the signs of the real parts of the eigenvalues of the matrix in Eq. 4.21 are not clear. However, using the asymptotic result (Eq. 4.19), for $\beta \ll 1$, we obtain the simpler matrix

$$
\left(\begin{array}{cc}
-\beta & \frac{\beta \xi}{\xi+\alpha \nu} \\
\left(\frac{\beta^{2} \varrho(\xi+\alpha \nu)}{12}\right)^{1 / 3}-\left(\frac{\beta^{2} \varrho(\xi+\alpha \nu)}{12}\right)^{1 / 3} & -\frac{\xi}{2}\left(\frac{2 \beta^{2} \varrho}{3(\xi+\alpha \nu)^{2}}\right)^{1 / 3} \\
\beta^{1 / 3}\left(\frac{\xi+\alpha \nu}{12 \varrho}\right)^{2 / 3} & -\frac{\xi}{2}\left(\frac{\beta \varrho^{2}}{18(\xi+\alpha \nu)}\right)^{1 / 3}-\mu \nu-\beta^{1 / 3}\left(\frac{\xi+\alpha \nu}{12 \varrho}\right)^{2 / 3}
\end{array}\right),
$$

whose characteristic polynomial is

$$
0=q^{3}+\mu \nu q^{2}+\mu \nu\left(\frac{1}{12} \beta^{2} \varrho(\xi+\alpha \nu)\right)^{1 / 3} q-D,
$$

Formally $D$ is the determinant of the matrix in Eq. 4.23, which is zero, giving a zero eigenvalue, which indicates marginal stability. Hence, we return to the more accurate matrix in Eq. 4.21, which gives $D \sim-\beta^{2} \mu \nu$. The polynomial (Eq. 4.24) thus has roots

$$
q_{1} \sim-\mu \nu, \quad q_{2} \sim-\left(\frac{\beta^{2} \varrho(\xi+\alpha \nu)}{12}\right)^{1 / 3}, \quad q_{3} \sim-\left(\frac{12 \beta^{4}}{\varrho(\alpha \nu+\xi)}\right)^{1 / 3} .
$$

This means that the symmetric state is always linearly stable for this asymptotic scaling. We expect to observe evolution on three distinct timescales, one of $\mathcal{O}(1)$, one of $\mathcal{O}\left(\beta^{-2 / 3}\right)$ and one of $\mathcal{O}\left(\beta^{-4 / 3}\right)$.

We now consider the other asymptotic limit, namely, $\alpha \sim \xi \gg 1$ and all other parameters are $\mathcal{O}(1)$. In this case, taking the leading order terms in each row, the stability matrix in Eq. 4.21 reduces to

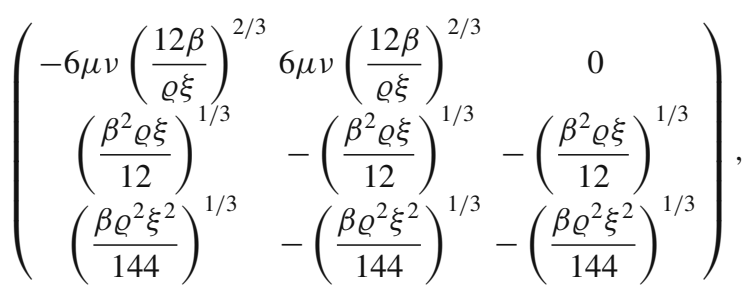

which again formally has a zero determinant. The characteristic polynomial is

$$
0=q^{3}+q^{2}+6 \beta \mu \nu q-D,
$$

wherein we again take the more accurate determinant obtained from a higher-order expansion of Eq. 4.21, namely $D=\beta^{2} \mu \nu$. The eigenvalues are then given by

$$
q_{1} \sim-\left(\frac{\beta \varrho^{2} \xi^{2}}{144}\right)^{1 / 3}, \quad q_{2,3} \sim \pm \sqrt{\beta \mu \nu}\left(\frac{12 \beta}{\varrho \xi}\right)^{1 / 3} .
$$




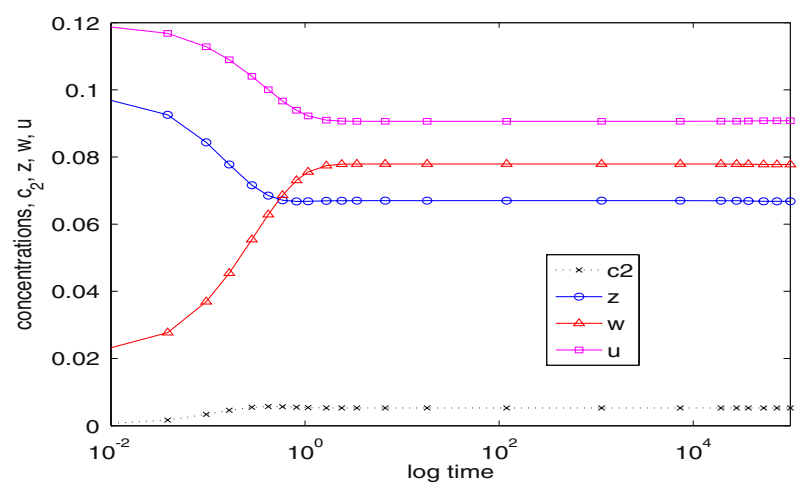

Fig. 10 Illustration of the evolution of the total concentrations $c_{2}, z, w, u$ for a numerical solution of the system truncated at hexamers (Eqs. 4.1-4.7) in the limit $\alpha \sim \xi \gg 1$. Since model equations are in nondimensional form, the time units are arbitrary. The parameters are $\alpha=\xi=30, v=0.5, \beta=$ $\mu=1$, and the initial data is $x_{6}(0)=y_{6}(0)=0.06, x_{4}(0)=y_{4}(0)=0.01, x_{2}(0)=0.051, y_{2}(0)=0.049$, $c_{2}(0)=0$. Note the time axis has a logarithmic scale

We now observe that there is always one stable and two unstable eigenvalues, so we deduce that the system breaks symmetry in the case $\alpha \sim \xi \gg 1$. The first eigenvalue corresponds to a faster timescale where $t \sim \mathcal{O}\left(\xi^{-2 / 3}\right)$ whilst the latter two correspond to the slow timescale where $t=\mathcal{O}\left(\xi^{1 / 3}\right)$.

\section{Simulation Results}

We briefly review the results of a numerical simulation of Eqs. 4.1-4.7 in the case $\alpha \sim \xi \gg 1$ to illustrate the symmetry-breaking observed therein. Although the numerical simulation used the variables $x_{k}$ and $y_{k}(k=2,4,6)$ and $c_{2}$, we plot the total concentrations $z, w, u$ in Fig. 10. The initial conditions have a slight imbalance in the handedness of small crystals $\left(x_{2}, y_{2}\right)$. The chiralities of small $\left(x_{2}, y_{2}, z\right)$, medium $\left(x_{4}, y_{4}, w\right)$, and larger $\left(x_{6}, y_{6}, u\right)$ are plotted in Fig. 11 on a log-log scale. Whilst Fig. 10 shows the concentrations in the system has equilibrated by $t=10$, at this stage the

Fig. 11 Graph of the evolution of the chiralities against time on a log-log scale; results of numerical simulation of the same hexamer-truncated system, with identical initial data and parameters as in Fig. 10

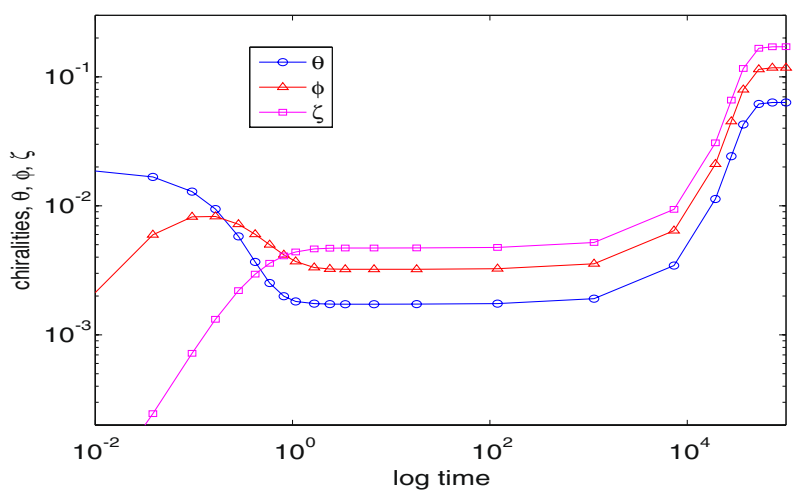


chiralities are in a metastable state, that is, a long plateau in the chiralities between $t=10$ and $t=10^{3}$ where little appears to change. There then follows a period of equilibration of chirality on the longer timescale when $t \sim 10^{4}$. We have observed this significant delay between the equilibration of concentrations and that of chiralities in a large number of simulations. The reason for this difference in timescales is due to the differences in the sizes of the eigenvalues in Eq. 4.25.

We have also investigated the case $\beta \ll 1$ with all other parameters $\mathcal{O}$ (1) to verify that this case does indeed approach the racemic state at large times (that is, $\theta, \phi, \zeta \rightarrow$ 0 as $t \rightarrow \infty)$. However, once again the difference in timescales can be observed, with the concentrations reaching equilibration on a faster timescale than the chiralities, due to the different magnitudes of eigenvalues (Eq. 4.28).

\section{New Simplifications of the System}

We return to the Eqs. 2.35-2.39 in the case $\delta=0$, now writing $x_{2}=x$ and $y=y_{2}$ to obtain

$$
\begin{aligned}
\frac{\mathrm{d} c}{\mathrm{~d} t} & =-2 \mu c+\mu \nu(x+y)-\alpha c\left(N_{x}+N_{y}\right), \\
\frac{\mathrm{d} x}{\mathrm{~d} t} & =\mu c-\mu v x-\alpha x c+\beta\left(N_{x}-x+x_{4}\right)-\xi x^{2}-\xi x N_{x}, \\
\frac{\mathrm{d} y}{\mathrm{~d} t} & =\mu c-\mu \nu y-\alpha y c+\beta\left(N_{y}-y+y_{4}\right)-\xi y^{2}-\xi y N_{y}, \\
\frac{\mathrm{d} N_{x}}{\mathrm{~d} t} & =\mu c-\mu \nu x+\beta\left(N_{x}-x\right)-\xi x N_{x}, \\
\frac{\mathrm{d} N_{y}}{\mathrm{~d} t} & =\mu c-\mu \nu y+\beta\left(N_{y}-y\right)-\xi y N_{y},
\end{aligned}
$$

which are not closed, since $x_{4}, y_{4}$ appear on the RHs's of Eqs. 5.2 and 5.3, hence we need to find formulae to determine $x_{4}$ and $y_{4}$ in terms of $x, y, N_{x}, N_{y}$.

One way of achieving this is to expand the system to include other properties of the distribution of cluster sizes. For example, equations governing the mass of crystals in each chirality can be derived as

$$
\frac{\mathrm{d} \varrho_{x}}{\mathrm{~d} t}=2 \mu c-2 \mu \nu x+2 \alpha c N_{x}, \quad \frac{\mathrm{d} \varrho_{y}}{\mathrm{~d} t}=2 \mu c-2 \mu \nu y+2 \alpha c N_{y} .
$$

These introduce no more new new quantities into the macroscopic system of equations, and do not rely on knowing $x_{4}$ or $y_{4}$, (although they do require knowledge of $x$ and $y$ ).

In the remainder of this section we consider various potential formulae for $x_{4}, y_{4}$ in terms of macroscopic quantities so that a macroscopic system can be constructed. We 
then analyse such macroscopic systems in two specific limits to show that predictions relating to symmetry-breaking can be made.

\section{Reductions}

The equations governing the larger cluster sizes $x_{k}, y_{k}$, are

$$
\frac{\mathrm{d} x_{2 k}}{\mathrm{~d} t}=\beta\left(x_{2 k+2}-x_{2 k}\right)-\left(x_{2 k}-x_{2 k-2}\right)(\alpha c+\xi x)
$$

in general this has solutions of the form $x_{2 k}=\sum_{j} A_{j}(t) \Lambda_{j}^{k-1}$, where $\Lambda_{j}$ are parameters (typically taking values between unity (corresponding to a steady-state in which mass is being added to the distribution) and $\frac{\alpha c+\xi x}{\beta}$ (the equilibrium value); and $A_{j}(t)$ are time-dependent; for some $\Lambda_{j}, A_{j}$ will be constant.

We assume that the distribution of each chirality of cluster is given by

$$
x_{2 k}=x\left(1-\frac{1}{\lambda_{x}}\right)^{k-1}, \quad y_{2 k}=y\left(1-\frac{1}{\lambda_{y}}\right)^{k-1}
$$

since solutions of this form may be steady-states of the governing Eq. 5.7. However, in our approximations for $x_{4}$ and $y_{4}$ the parameters $\lambda_{x}, \lambda_{y}$ are permitted to vary with time in some way that depends on other quantities in the model equations. The resulting expressions for the macroscopic number and mass quantities are

$$
\begin{array}{cc}
N_{x}=\sum_{k=1}^{\infty} x_{2 k}=x \lambda_{x}, & N_{y}=\sum_{k=1}^{\infty} y_{2 k}=y \lambda_{y}, \\
\varrho_{x}=\sum_{k=1}^{\infty} 2 k x_{2 k}=2 x \lambda_{x}^{2}, & \varrho_{y}=\sum_{k=1}^{\infty} 2 k y_{2 k}=2 y \lambda_{y}^{2} .
\end{array}
$$

Our aim is to find a simpler expression for the terms $x_{4}$ and $y_{4}$ which occur in Eqs. 5.2 and 5.3 , these are given by $x_{4}=x\left(1-1 / \lambda_{x}\right)$ where

$$
\lambda_{x}=\frac{N_{x}}{x}=\frac{\varrho_{x}}{2 N_{x}}=\sqrt{\frac{\varrho_{x}}{2 x}}
$$

hence

$$
x_{4}=x-\frac{x^{2}}{N_{x}}, \quad x_{4}=x-\frac{2 x N_{x}}{\varrho_{x}}, \quad \text { or } \quad x_{4}=x-x \sqrt{\frac{2 x}{\varrho_{x}}} .
$$

There are thus three possible reductions of the Eqs. 5.1-5.5, each eliminating one of $x, N_{x}, \varrho_{x}$ (and the corresponding $y, N_{y}, \varrho_{y}$ ). We consider each reduction in turn in the following subsections. Since some of these reductions involve $\varrho_{x}, \varrho_{y}$, we also use the evolution Eq. 5.6 for these quantities. 
Reduction 1: to $x, y, N_{x}, N_{y}$

Here we assume $\lambda_{x}=N_{x} / x, \lambda_{y}=N_{y} / y$, so, in addition to Eqs. 5.1, 5.4-5.5 the equations are

$$
\begin{aligned}
& \frac{\mathrm{d} x}{\mathrm{~d} t}=\mu c-\mu \nu x+\beta N_{x}-\frac{\beta x^{2}}{N_{x}}-\xi x^{2}-\xi x N_{x}, \\
& \frac{\mathrm{d} y}{\mathrm{~d} t}=\mu c-\mu \nu y+\beta N_{y}-\frac{\beta y^{2}}{N_{y}}-\xi y^{2}-\xi y N_{y} ;
\end{aligned}
$$

we have no need of the densities $\varrho_{x}, \varrho_{y}$ in this formulation.

The disadvantage of this reduction is that, due to Eq. 5.11, the total mass is given by

$$
\varrho=2 c+\varrho_{x}+\varrho_{y}=2 c+\frac{2 N_{x}^{2}}{x}+\frac{2 N_{y}^{2}}{y},
$$

and there is no guarantee that this will be conserved.

We once again consider the system in terms of total concentrations and relative chiralities by applying the transformation

$$
x=\frac{1}{2} z(1+\theta), \quad y=\frac{1}{2} z(1-\theta), \quad N_{x}=\frac{1}{2} N(1+\phi), \quad N_{y}=\frac{1}{2} N(1-\phi),
$$

to obtain the equations

$$
\begin{aligned}
\frac{\mathrm{d} c}{\mathrm{~d} t}= & -2 \mu c+\mu \nu z-\alpha c N \\
\frac{\mathrm{d} z}{\mathrm{~d} t}= & 2 \mu c-\mu \nu z-\alpha c z+\beta N-\frac{\beta z^{2}\left(1+\theta^{2}-2 \theta \phi\right)}{N\left(1-\phi^{2}\right)} \\
& -\frac{1}{2} \xi z^{2}\left(1+\theta^{2}\right)-\frac{1}{2} \xi z N(1+\theta \phi), \\
\frac{\mathrm{d} N}{\mathrm{~d} t}= & 2 \mu c-\mu \nu z+\beta N-\beta z-\frac{1}{2} \xi z N(1+\theta \phi) . \\
\frac{\mathrm{d} \theta}{\mathrm{d} t}= & -\left(\mu \nu+\alpha c+\xi z+\frac{1}{2} \xi N+\frac{2 \beta z}{N\left(1-\phi^{2}\right)}+\frac{1}{z} \frac{\mathrm{d} z}{\mathrm{~d} t}\right) \theta \\
& +\left(\frac{\beta N}{z}-\frac{1}{2} \xi N+\frac{\beta z\left(1+\theta^{2}\right)}{N\left(1-\phi^{2}\right)}\right) \phi, \\
\frac{\mathrm{d} \phi}{\mathrm{d} t}= & -\left(\mu \nu+\beta+\frac{1}{2} \xi N\right) \frac{z}{N} \theta+\left(\beta-\frac{1}{2} \xi z-\frac{1}{N} \frac{\mathrm{d} N}{\mathrm{~d} t}\right) \phi .
\end{aligned}
$$

These equations have the symmetric steady-state given by $\theta=0=\phi$ and $c, z, N$ satisfying

$$
c=\frac{\mu \nu z}{2 \mu+\alpha N}, \quad z=\frac{2 \beta N(2 \mu+\alpha N)}{(2 \beta+\xi N)(2 \mu+\alpha N)+2 \alpha \mu \nu N},
$$


from Eqs. 5.17 and 5.19. Note that the steady state value of $N$ will depend upon the initial conditions, it is not determined by Eq. 5.18. This is because the steadystate equations obtained by setting the time derivatives in Eqs. 5.17-5.19 are not independent. The difference (Eqs. 5.18 and 5.19) is equal to $z / N$ times the sum (Eqs. $5.17+5.19)$.

In "Asymptotic Limit 1: $\beta \ll 1$ ” and "Asymptotic Limit 2: $\alpha \sim \xi \gg 1$ ” below, so as to discuss the stability of a solution in the two asymptotic regimes $\beta \ll 1$ and $\alpha \sim$ $\xi \gg 1$, we augment the steady-state Eqs. 5.17-5.19 with the condition $\varrho=2 N^{2} / z$, with $\varrho$ assumed to be $\mathcal{O}(1)$.

The linear stability of $\theta=0=\phi$ is given by assuming $\theta$ and $\phi$ are small, yielding the system

$$
\frac{\mathrm{d}}{\mathrm{d} t}\left(\begin{array}{c}
\theta \\
\phi
\end{array}\right)=\left(\begin{array}{cc}
-\left(\frac{2 \mu c}{z}+\frac{\xi z}{2}+\frac{\beta z}{N}+\frac{\beta N}{z}\right) & \left(\frac{\beta N}{z}+\frac{\beta z}{N}-\frac{\xi N}{2}\right) \\
-\left(\mu \nu+\beta+\frac{1}{2} \xi N\right) \frac{z}{N} & \left(\beta+\mu \nu-\frac{2 \mu c}{z}\right) \frac{z}{N}
\end{array}\right)\left(\begin{array}{l}
\theta \\
\phi
\end{array}\right) .
$$

An instability of the symmetric solution is indicated by the determinant of this matrix being negative. Substituting Eq. 5.22 into the determinant, yields

$$
\operatorname{det}=\frac{\beta \mu \nu\left(4 \beta \mu-\alpha \xi N^{2}\right)}{4 \beta \mu+2 \alpha \beta N+2 \mu \xi N+2 \alpha \mu \nu N+\alpha \xi N^{2}} .
$$

Hence we find that the symmetric (racemic) state is unstable if $N>2 \sqrt{\mu \beta / \alpha \xi}$, that is, large aggregation rates $(\alpha, \xi)$ and slow grinding $(\beta)$ are preferable for symmetrybreaking.

We consider two specific asymptotic limits of parameter values so as to derive specific results for steady-states and conditions on stability. In both limits, we have that the aggregation rates dominate fragmentation $(\alpha \sim \xi \gg \beta)$, so that the system is strongly biased towards the formation of crystals and the dimer concentrations are small. In the first case we assume that the fragmentation is small and the aggregation rates are of a similar scale to the interconversion of dimers $(\beta \ll \mu \sim \alpha \sim \xi=\mathcal{O}(1))$; whilst the second has a fragmentation rate of similar size to the dimer conversion rates and larger aggregation rates $(\alpha \sim \xi \gg \mu \sim \beta=\mathcal{O}(1))$.

Asymptotic Limit 1: $\beta \ll 1$

In the case of asymptotic limit $1, \beta \ll 1$, we find the steady-state solution

$$
N \sim \sqrt{\frac{\beta \varrho}{\xi+\alpha \nu}}, \quad z \sim \frac{2 \beta}{\xi+\alpha \nu}, \quad c \sim \frac{\beta \nu}{\xi+\alpha \nu} .
$$

From Eq. 5.24, we find an instability if $\varrho>\varrho_{c}:=4 \mu(\xi+\alpha \nu) / \alpha \xi$. That is, larger masses $(\varrho)$ favour symmetry-breaking, as do larger aggregation rates $(\alpha, \xi)$. The eigenvalues of Eq. 5.23 in this limit are $q_{1}=-\mu \nu-$ a fast stable mode of the dynamics and

$$
q_{2}=\frac{\alpha \xi \beta^{3 / 2}}{2 \mu \sqrt{\varrho}(\xi+\alpha \nu)^{3 / 2}}\left(\varrho-\frac{4 \mu(\xi+\alpha \nu)}{\alpha \xi}\right),
$$

which indicates a slowly growing instability when $\varrho>\varrho_{c}$. Hence the balace of achiral to chiral morphologies of smaller clusters $(v)$ also influences the propensity for 
non-racemic solution. However, since the dynamics described by this model does not conserve total mass, the results from this should be treated with some caution, and we now analyse models which do conserve total mass.

Asymptotic Limit 2: $\alpha \sim \xi \gg 1$

In this case we find the steady-state solution is given by

$$
N \sim \sqrt{\frac{\beta \varrho}{\xi}}, \quad z \sim \frac{2 \beta}{\xi}, \quad c \sim \frac{4 \mu \nu}{\alpha} \sqrt{\frac{\beta}{\xi \varrho}} .
$$

The condition following from Eq. 5.24 then implies that we have an instability if $\varrho>$ $\varrho_{c}:=4 \mu / \alpha \ll 1$. The eigenvalues of the stability matrix are $q_{1}=-\frac{1}{2} \sqrt{\beta \varrho \xi}$, which is large and negative, indicating attraction to some lower dimensional solution over a relatively fast timescale; the eigenvector being $(1,0)^{T}$ showing that $\theta \rightarrow 0$. The other eigenvalue is $q_{2}=2 \mu \nu \sqrt{\beta / \varrho \xi} \ll 1$, and corresponds to a slow growth of the chirality of the solution, since it relates to the eigenvector $(0,1)^{T}$. Assuming the system is initiated near its symmetric solution $(\theta=\phi=0)$, this shows that the distribution of clusters changes its chirality first, whilst the dimer concentrations remain, at least to leading order, racemic. We expect that at a later stage the chirality of the dimers too will become nonzero.

Reduction 2: to $x, y, \varrho_{x}, \varrho_{y}$

Here we eliminate $x_{4}=x\left(1-1 / \lambda_{x}\right), y_{4}=y\left(1-1 / \lambda_{y}\right)$ together with $N_{x}$ and $N_{y}$ using

$$
\lambda_{x}=\sqrt{\frac{\varrho_{x}}{2 x}}, \quad \lambda_{y}=\sqrt{\frac{\varrho_{y}}{2 y}}, \quad N_{x}=\sqrt{\frac{x \varrho_{x}}{2}}, \quad N_{y}=\sqrt{\frac{y \varrho_{y}}{2}},
$$

leaving a system of equations for $\left(c, x, y, \varrho_{x}, \varrho_{y}\right)$

$$
\begin{aligned}
\frac{\mathrm{d} c}{\mathrm{~d} t} & =\mu \nu(x+y)-2 \mu c-\sqrt{2} \alpha c\left(\sqrt{x \varrho_{x}}+\sqrt{y \varrho_{y}}\right), \\
\frac{\mathrm{d} x}{\mathrm{~d} t} & =\mu c-\mu \nu x-\alpha c x-\xi x^{2}-\xi x \sqrt{\frac{x \varrho_{x}}{2}}+\beta \sqrt{\frac{x \varrho_{x}}{2}}-\beta x \sqrt{\frac{2 x}{\varrho_{x}}}, \\
\frac{\mathrm{d} \varrho_{x}}{\mathrm{~d} t} & =-2 \mu \nu x+2 \mu c+2 \alpha c \sqrt{\frac{x \varrho_{x}}{2}}
\end{aligned}
$$

with similar equations for $y, \varrho_{y}$. Transforming to total concentrations and relative chiralities by way of

$$
x=\frac{1}{2} z(1+\theta), \quad y=\frac{1}{2} z(1-\theta), \quad \varrho_{x}=\frac{1}{2} R(1+\zeta), \quad \varrho_{y}=\frac{1}{2} R(1-\zeta),
$$


we find

$$
\begin{aligned}
\frac{\mathrm{d} c}{\mathrm{~d} t}= & \mu \nu z-2 \mu c-\frac{\alpha c \sqrt{z R}}{2 \sqrt{2}}[\sqrt{(1+\theta)(1+\zeta)}+\sqrt{(1-\theta)(1-\zeta)}] \\
\frac{\mathrm{d} z}{\mathrm{~d} t}= & 2 \mu c-\mu \nu z-\alpha c z-\frac{1}{2} \xi z^{2}\left(1+\theta^{2}\right) \\
& +\frac{\beta \sqrt{z R}}{2 \sqrt{2}}[\sqrt{(1+\theta)(1+\zeta)}+\sqrt{(1-\theta)(1-\zeta)}] \\
& -\frac{\xi z^{3 / 2} R^{1 / 2}}{4 \sqrt{2}}\left[(1+\theta)^{3 / 2}(1+\zeta)^{1 / 2}+(1-\theta)^{3 / 2}(1-\zeta)^{1 / 2}\right] \\
& -\frac{\beta z^{3 / 2}}{\sqrt{2 R}}\left[\frac{(1+\theta)^{3 / 2}}{(1+\zeta)^{1 / 2}}+\frac{(1-\theta)^{3 / 2}}{(1-\zeta)^{1 / 2}}\right] \\
\frac{\mathrm{d} R}{\mathrm{~d} t}= & -2 \mu \nu z+4 \mu c+\frac{1}{2} \alpha c \sqrt{2 z R}[\sqrt{(1+\theta)(1+\zeta)}+\sqrt{(1-\theta)(1-\zeta)}]
\end{aligned}
$$

together with the Eqs. 5.38 and 5.39 for the relative chiralities $\theta$ and $\zeta$, which will be analysed later.

Since the equations for $\mathrm{d} R / d d t$ and $\mathrm{d} c / \mathrm{d} t$ are essentially the same, we obtain a third piece of information from the requirement that the total mass in the system is unchanged from the initial data, hence the new middle equation above. Solving these we find $c=\frac{1}{2}(\varrho-R)$ and use this in place of the equation for $c$.

In the symmetric case $(\theta=\zeta=0)$ we obtain the steady-state conditions

$$
\begin{aligned}
& 0=2 \mu \nu z-4 \mu c-\alpha c \sqrt{2 z R}, \quad \varrho=R+2 c, \\
& 0=2 \mu c-\mu \nu z-\alpha c z-\frac{1}{2} \xi z^{2}+\frac{1}{2} \beta \sqrt{2 z R}-\beta z \sqrt{\frac{2 z}{R}}-\frac{\xi z}{2} \sqrt{\frac{z R}{2}} .
\end{aligned}
$$

For small $\theta, \zeta$, the equations for the chiralities can be approximated by

$$
\begin{aligned}
\frac{\mathrm{d} \theta}{\mathrm{d} t}= & -\left(\frac{2 \mu c}{z}+\frac{1}{2} \xi z+\frac{1}{2} \beta \sqrt{\frac{R}{2 z}}+\frac{1}{2} \beta \sqrt{\frac{2 z}{R}}+\frac{1}{4} \xi \sqrt{\frac{z R}{2}}\right) \theta \\
& +\left(\frac{\beta(R+2 z)}{2 \sqrt{2 z R}}-\frac{\xi}{4} \sqrt{\frac{R z}{2}}\right) \zeta, \\
\frac{\mathrm{d} \zeta}{\mathrm{d} t}= & \left(\frac{2 \mu \nu z}{R}-\alpha c \sqrt{\frac{z R}{2}}\right) \theta-\left(\frac{2 \mu \nu z}{R}-\frac{4 \mu c}{R}\right) \zeta,
\end{aligned}
$$

We analyse the stability of the symmetric (racemic) state in the two limits $\beta \ll 1$ and $\alpha \sim \xi \gg 1$ in the next subsections.

\section{Asymptotic Limit 1: $\beta \ll 1$}

In this case, solving the conditions (Eqs. 5.36 and 5.37) asymptotically, we find

$$
z \sim \frac{2 \beta}{\xi+\alpha \nu}, \quad c \sim \frac{\beta \nu}{\xi+\alpha \nu}, \quad R \sim \varrho-2 c .
$$


Substituting these values into the differential equations which determine the stability of the racemic state leads to

$$
\frac{\mathrm{d}}{\mathrm{d} t}\left(\begin{array}{l}
\theta \\
\zeta
\end{array}\right)\left(\begin{array}{cc}
-\mu \nu & \frac{\alpha \nu}{4} \sqrt{\frac{\beta \varrho}{\xi+\alpha \nu}} \\
-\frac{4 \beta \mu \nu}{\varrho(\xi+\alpha \nu)} & \frac{\alpha \nu \beta^{3 / 2}}{(\xi+\alpha \nu)^{3 / 2} \sqrt{\varrho}}
\end{array}\right)\left(\begin{array}{l}
\theta \\
\zeta
\end{array}\right) .
$$

Formally this matrix has eigenvalues of zero and $-\mu \nu$. Since the zero eigenvalue indicates marginal stability of the racemic solution, we need to consider higher-order terms to obtain a more definite result.

Going to higher order, gives the determinant of the resulting matrix as $-\alpha \xi \nu /(\alpha \nu+\xi)^{2}$ hence the eigenvalues are

$$
q_{1}=-\mu \nu, \quad \text { and } \quad q_{2}=\frac{\alpha \xi}{\mu(\alpha \nu+\xi)^{2}}
$$

the former indicating a rapid decay of $\theta$ (corresponding to the eigenvector $(1,0)^{T}$ ), and the latter showing a slow divergence from the racemic state in the $\zeta$-direction, at leading order, according to

$$
\left(\begin{array}{l}
\theta \\
\zeta
\end{array}\right) \sim C_{1}\left(\begin{array}{l}
0 \\
1
\end{array}\right) \exp \left(\frac{\alpha \xi t}{\mu(\alpha \nu+\xi)^{2}}\right) .
$$

Hence in the case $\beta \ll 1$, we find an instability of the symmetric solution for all other parameter values.

Asymptotic Limit 2: $\alpha \sim \xi \gg 1$

In this case, solving the conditions (Eqs. 5.36 and 5.37) asymptotically, we find

$$
z \sim \frac{2 \beta}{\xi}, \quad c \sim \frac{2 \mu \nu}{\alpha} \sqrt{\frac{\beta}{\varrho \xi}}, \quad R \sim \varrho-2 c .
$$

Substituting these values into the differential Eqs. 5.38 and 5.39 which determine the stability of the racemic state leads to

$$
\frac{\mathrm{d}}{\mathrm{d} t}\left(\begin{array}{l}
\theta \\
\zeta
\end{array}\right)\left(\begin{array}{cc}
-\frac{1}{2} \sqrt{\beta \xi \varrho} & o(\sqrt{\xi}) \\
-\frac{4 \beta \mu \nu}{\varrho \xi} & \frac{4 \beta \mu \nu}{\varrho \xi}
\end{array}\right)\left(\begin{array}{l}
\theta \\
\zeta
\end{array}\right)
$$

hence the eigenvalues are $q_{1}=-\frac{1}{2} \sqrt{\beta \varrho \xi}$ and $q_{2}=4 \mu \nu \beta / \varrho \xi$, (in the above $o(\sqrt{\xi}$ ) means a quantity $q$ satisfying $q \ll \sqrt{\xi}$ as $\xi \rightarrow \infty$ ). Whilst the former indicates the existence of a stable manifold (with a fast rate of attraction), the latter shows that there is also an unstable manifold. Although the timescale associated with this is much slower, it shows that the symmetric (racemic) state is unstable. 
Reduction 3: to $N_{x}, N_{y}, \varrho_{x}, \varrho_{y}$

In this case our aim is to retain only information on the number and typical size of crystal distribution, so we eliminate the dimer concentrations $x, y$, using

$$
\lambda_{x}=\frac{\varrho_{x}}{2 N_{x}}, \quad \lambda_{y}=\frac{\varrho_{y}}{2 N_{y}}, \quad x=\frac{2 N_{x}^{2}}{\varrho_{x}}, \quad y=\frac{2 N_{y}^{2}}{\varrho_{y}} .
$$

These transformations reformulate the governing Eqs. 5.1-5.6 to

$$
\begin{aligned}
\frac{\mathrm{d} N_{x}}{\mathrm{~d} t} & =\frac{1}{2} \mu(\varrho-R)+\beta N_{x}-2(\mu \nu+\beta) \frac{N_{x}^{2}}{\varrho_{x}}-\frac{2 \xi N_{x}^{3}}{\varrho_{x}}, \\
\frac{\mathrm{d} N_{y}}{\mathrm{~d} t} & =\frac{1}{2} \mu(\varrho-R)+\beta N_{y}-2(\mu \nu+\beta) \frac{N_{y}^{2}}{\varrho_{y}}-\frac{2 \xi N_{y}^{3}}{\varrho_{y}}, \\
\frac{\mathrm{d} \varrho_{x}}{\mathrm{~d} t} & =(\varrho-R)\left(\mu+\alpha N_{x}\right)-\frac{4 \mu \nu N_{x}^{2}}{\varrho_{x}}, \\
\frac{\mathrm{d} \varrho_{y}}{\mathrm{~d} t} & =(\varrho-R)\left(\mu+\alpha N_{y}\right)-\frac{4 \mu \nu N_{y}^{2}}{\varrho_{y}},
\end{aligned}
$$

where $R:=\varrho_{x}+\varrho_{y}$. We now transform to total concentrations $(N, R)$ and relative chiralities $(\phi$ and $\zeta)$ via

$$
N_{x}=\frac{1}{2} N(1+\phi), \quad N_{y}=\frac{1}{2} N(1-\phi), \quad \varrho_{x}=\frac{1}{2} R(1+\zeta), \quad \varrho_{y}=\frac{1}{2} R(1-\zeta),
$$

together with $c=\frac{1}{2}(\varrho-R)$, to obtain

$$
\begin{aligned}
\frac{\mathrm{d} R}{\mathrm{~d} t}= & (\varrho-R)(2 \mu+\alpha N)-\frac{4 \mu \nu N^{2}\left(1+\phi^{2}-2 \phi \zeta\right)}{R\left(1-\zeta^{2}\right)} \\
\frac{\mathrm{d} N}{\mathrm{~d} t}= & \mu(\varrho-R)+\beta N \\
& -\frac{N^{2}}{R\left(1-\zeta^{2}\right)}\left[2(\mu \nu+\beta)\left(1+\phi^{2}-2 \phi \zeta\right)+\xi N\left(1+3 \phi^{2}-3 \phi \zeta-\phi^{3} \zeta\right)\right] \\
\frac{\mathrm{d} \phi}{\mathrm{d} t}= & \beta \phi-\frac{1}{N} \frac{\mathrm{d} N}{\mathrm{~d} t} \phi \\
& -\frac{N}{R\left(1-\zeta^{2}\right)}\left[2(\beta+\mu \nu)\left(2 \phi-\zeta-\phi^{2} \zeta\right)+\xi N\left(3 \phi-\zeta+\phi^{3}-3 \phi^{2} \zeta\right)\right] \\
\frac{\mathrm{d} \zeta}{\mathrm{d} t}= & \frac{\alpha(\varrho-R) N \phi}{R}-\frac{1}{R} \frac{\mathrm{d} R}{\mathrm{~d} t} \zeta-\frac{4 \mu \nu N^{2}\left(2 \phi-\zeta-\phi^{2} \zeta\right)}{R^{2}\left(1-\zeta^{2}\right)}
\end{aligned}
$$

We now analyse this system in more detail, since this set of equations conserves mass, and is easier to analyse than Eqs. 5.33-5.35 due to the absence of square roots. We consider the two asymptotic limits $(\beta \ll 1$ and $\alpha \sim \xi \gg 1)$ in which, at steady-state, the majority of mass is in the form of clusters. 
The Symmetric Steady-State

Putting $\zeta=0=\phi$, we find the symmetric steady-state is given by

$$
\begin{aligned}
& 0=(\varrho-R)(2 \mu+\alpha N)-\frac{4 \mu \nu N^{2}}{R}, \\
& 0=\mu(\varrho-R)+\beta N-2(\mu \nu+\beta) \frac{N^{2}}{R}-\frac{\xi N^{3}}{R} .
\end{aligned}
$$

the former is solved by one of

$$
\begin{gathered}
R=\frac{1}{2} \varrho\left(1 \pm \sqrt{1-\frac{16 \mu \nu N^{2}}{(2 \mu+\alpha N) \varrho^{2}}}\right), \\
N=\frac{\alpha R(\varrho-R)}{8 \mu \nu}\left(1+\sqrt{1+\frac{32 \mu^{2} v}{\alpha^{2} R(\varrho-R)}}\right) .
\end{gathered}
$$

More complete asymptotic solutions will be derived in the sections titled “Asymptotic Limit 1: $\beta \ll 1$ " and "Asymptotic Limit 2: $\alpha \sim \xi \gg 1$ ".

\section{Stability of the Symmetric State}

We now consider the stability of the symmetric steady-state. For small $\phi, \zeta$ we have

$$
\frac{R}{N} \frac{\mathrm{d}}{\mathrm{d} t}\left(\begin{array}{l}
\phi \\
\zeta
\end{array}\right)=\left(\begin{array}{cc}
-2 \beta-2 \mu \nu-2 \xi N-\frac{\mu(\varrho-R) R}{N^{2}} & 2 \beta+2 \mu \nu+\xi N \\
\left(\alpha(\varrho-R)-\frac{8 \mu \nu N}{R}\right)^{2} & 8 \mu \nu-\frac{(\varrho-R)(2 \mu+\alpha N) R}{N^{2}}
\end{array}\right)\left(\begin{array}{l}
\phi \\
\zeta
\end{array}\right),
$$

and this is unstable if the determinant of this matrix is negative. Now we consider the two asymptotic limits in more detail.

\section{Asymptotic Limit 1: $\beta \ll 1$}

When fragmentation is slow, that is, $\beta \ll 1$, at steady-state we have $N=\mathcal{O}(\sqrt{\beta})$ and $R=\varrho-\mathcal{O}(\beta)$. Balancing terms in Eqs. 5.56 and 5.57 we find the same leading order equation twice, namely $2 v N^{2}=\beta \varrho(\varrho-R)$. Taking the difference of the two yields an independent equation from higher order terms, hence we obtain

$$
N \sim \sqrt{\frac{\beta \varrho}{\xi+\alpha \nu}}, \quad R \sim \varrho-\frac{2 \nu \beta}{\xi+\alpha \nu} .
$$

Note that this result implies that the dimer concentrations are small, with $c \sim z$ and $c \sim \beta \nu /(\xi+\alpha \nu), z \sim 2 \beta /(\xi+\alpha \nu)$. 
Substituting these expressions into those for the stability of the symmetric steadystate (Eq. 5.60), we find

$$
\frac{R}{4 \mu \nu N} \frac{\mathrm{d}}{\mathrm{d} t}\left(\begin{array}{l}
\phi \\
\zeta
\end{array}\right)=\left(\begin{array}{cc}
-1 & \frac{1}{2} \\
-2 \sqrt{\frac{\beta}{\varrho(\xi+\alpha \nu)}} & 1
\end{array}\right)\left(\begin{array}{l}
\phi \\
\zeta
\end{array}\right) .
$$

This matrix has one stable eigenvalue (corresponding to $(1,0)^{T}$ and hence the decay of $\phi$ whilst $\zeta$ remains invariant), the unstable eigenvector is $(1,4)^{T}$, hence we find

$$
\left(\begin{array}{l}
\phi(t) \\
\zeta(t)
\end{array}\right) \sim C\left(\begin{array}{l}
1 \\
4
\end{array}\right) \exp \left(\frac{4 \mu \nu t \sqrt{\beta}}{\sqrt{\varrho(\xi+\alpha \nu)}}\right)
$$

If we compare the timescale of this solution to that over which the concentrations $N, R$ vary, we find that symmetry-breaking occurs on a slower timescale than the evolution of cluster masses and numbers. This is illustrated in the numerical simulation of Eqs. 5.47-5.50 shown in Fig. 12. More specifically, the time-scale increases with the mass in the system, and with the ratio of aggregation to fragmentation rates, $(\alpha \nu+\xi) / \beta$, and is inversely related to the chiral switching rate of small clusters $(\mu \nu)$.

\section{Asymptotic Limit 2: $\alpha \sim \xi \gg 1$}

In this case we retain the assumptions that $\mu, v=\mathcal{O}(1)$, however, we now impose $\beta=\mathcal{O}(1)$ and $\alpha \sim \xi \gg 1$. For a steady-state, we require the scalings $N=\mathcal{O}(1 / \sqrt{\xi})$ and $\varrho-R=\mathcal{O}\left(1 / \xi^{3 / 2}\right)$. Specifically, solving Eqs. 5.56 and 5.57 we find

$$
N \sim \sqrt{\frac{\beta \varrho}{\xi}}, \quad R \sim \varrho-\frac{4 \mu \nu}{\alpha \varrho} \sqrt{\frac{\beta \varrho}{\xi}},
$$

hence the dimer concentrations $c=\frac{1}{2}(\varrho-R) \sim N^{3}=\mathcal{O}\left(1 / \xi^{3 / 2}\right)$ and $z=2 N^{2} / \varrho \sim$ $N^{2}=\mathcal{O}(1 / \xi)$. More precisely, $c \sim(2 \mu \nu / \alpha) \sqrt{\beta / \varrho \xi}$ and $z \sim 2 \beta / \xi$, in contrast with the previous asymptotic scaling which gave $z \sim N^{2}$ ).

Fig. 12 Graph of concentrations

$N_{x}, N_{y}, \varrho_{x}, \varrho_{y}, c$ against time on a logarithmic time for the asymptotic limit 1 , with initial conditions $N_{x}=0.2=N_{y}$, $\varrho_{x}=0.45, \varrho_{y}=0.44$, other parameters given by $\alpha=1=\xi=\mu, \beta=0.01$, $\varrho=8$. Since model equations are in nondimensional form, the time units are arbitrary

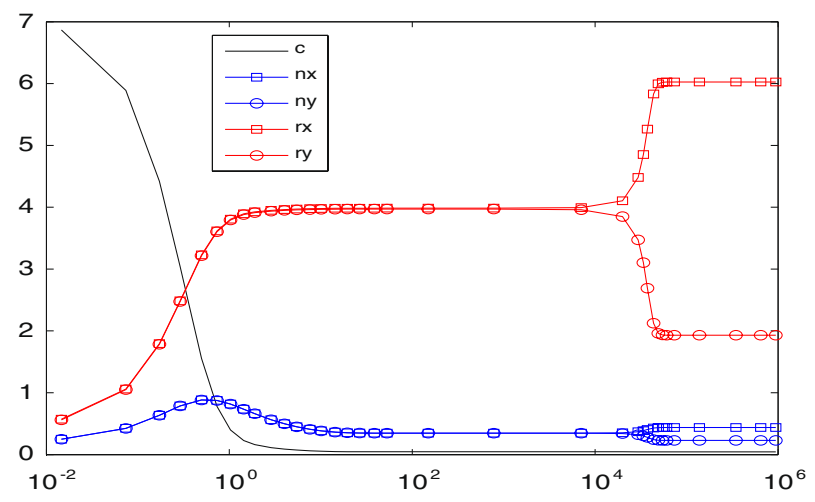


To determine the timescales for crystal growth and dissolution, we use Eq. 5.64 to define

$$
N \sim n(t) \sqrt{\beta \varrho / \xi}, \quad R \sim \varrho-\frac{4 \mu \nu r(t)}{\alpha \varrho} \sqrt{\frac{\beta \varrho}{\xi}},
$$

and so rewrite the governing Eqs. 5.52 and 5.53 as

$$
\begin{aligned}
& \frac{\mathrm{d} n}{\mathrm{~d} t}=\beta n\left(1-n^{2}-\frac{2 n(\beta+\mu \nu)}{\sqrt{\varrho \xi \beta}}\right), \\
& \frac{\mathrm{d} r}{\mathrm{~d} t}=\alpha \sqrt{\frac{\beta \varrho}{\xi}}\left(n^{2}-r-\frac{2 \mu r}{\alpha} \sqrt{\frac{\xi}{\beta \varrho}}\right) .
\end{aligned}
$$

Here, the former equation for $n(t)$ corresponds to the slower timescale, with a rate $\beta$, the rate of equilibration of $r(t)$ being $\alpha \sqrt{\beta \varrho / \xi}$.

The stability of the symmetric state is determined by

$$
\frac{R}{N} \frac{\mathrm{d}}{\mathrm{d} t}\left(\begin{array}{c}
\phi(t) \\
\zeta(t)
\end{array}\right)=\left(\begin{array}{cc}
-2 \sqrt{\beta \varrho \xi} & \sqrt{\beta \varrho \xi} \\
-4 \mu \nu \sqrt{\beta / \xi \varrho} & 4 \mu \nu
\end{array}\right)\left(\begin{array}{l}
\phi \\
\zeta
\end{array}\right)
$$

This matrix has one large negative eigenvalue $(\sim-2 \sqrt{\beta \varrho \xi})$ and one (smaller) positive eigenvalue $(\sim 4 \mu \nu)$; the former corresponds to $(1,0)^{T}$ hence the decay of $\phi$, whilst the latter corresponds to the eigenvector $(1,2)^{T}$. Hence the system (Eq. 5.68) has the solution

$$
\left(\begin{array}{l}
\phi \\
\zeta
\end{array}\right) \sim C\left(\begin{array}{l}
1 \\
2
\end{array}\right) \exp \left(4 \mu v t \sqrt{\frac{\beta}{\varrho \xi}}\right)
$$

The chiralities evolve on two timescales, the faster being $2 \beta$ corresponding to the stable eigenvalue of Eq. 5.68 and the slower unstable rate being $4 \mu \nu \sqrt{\beta / \xi \varrho}$. This timescale is similar to Eq. 5.63, being dependent on mass and the ratio of aggregation to fragmentation, and inversely proportional to the chiral switching rate of dimers $(\mu \nu)$. This case is illustrated in Figure 13.

The Asymmetric Steady-State

Since the symmetric state can be unstable, there must be some other large-time asymmetric attractor(s) for the system, which we now aim to find. From Eqs. 5.47 and 5.49, at steady-state, we have

$$
2 c_{2}\left(2 \mu+\alpha N_{x}\right)=\frac{4 \mu \nu N_{x}^{2}}{\varrho_{x}}, \quad \mu c_{2}+\beta N_{x}=2\left(\mu \nu+\beta+\xi N_{x}\right) \frac{N_{x}^{2}}{\varrho_{x}} .
$$

Taking the ratio of these we find a single quadratic equation for $N_{x}$

$$
0=\alpha \xi N_{x}^{2}-\left(\frac{\beta \mu \nu}{c_{2}}-\alpha \beta-\alpha \mu \nu-\xi \mu\right) N_{x}+\beta \mu,
$$


with an identical one for $N_{y}$. Hence there is the possibility of distinct solutions for $N_{x}$ and $N_{y}$ if both roots of Eq. 5.71 are positive; this occurs if

$$
c_{2}<\frac{\beta \mu \nu}{\alpha \beta+\xi \mu+\alpha \mu \nu+2 \sqrt{\alpha \beta \xi \mu}} .
$$

Given $N_{x}\left(N_{y}\right)$, we then have to solve one of Eq. 5.70 to find $\varrho_{x}\left(\varrho_{y}\right)$, via

$$
\varrho_{x}=\frac{2 \mu \nu N_{x}^{2}}{c_{2}\left(\mu+\alpha N_{x}\right)},
$$

and then satisfy the consistency condition that $\varrho_{x}+\varrho_{y}+2 c_{2}=\varrho$. After some algebra, this condition reduces to

$$
\begin{aligned}
\frac{1}{2} \alpha^{2} \xi c_{2}^{2}\left(\beta-\alpha c_{2}\right)\left(\varrho-2 c_{2}\right)= & \beta^{2} \mu^{2} \nu^{2}-\beta \mu \nu c_{2}[\alpha \beta+2 \alpha \mu \nu+2 \xi \mu] \\
& +\mu c_{2}^{2}\left[\mu(\alpha \nu+\xi)^{2}+\alpha \beta(\alpha \nu-\xi)\right] .
\end{aligned}
$$

Being a cubic, it is not straightforward to write down explicit solutions of this equation, hence we once again consider the two asymptotic limits $(\beta \ll 1$ and $\alpha \sim$ $\xi \gg 1)$.

Asymptotic Limit 1: $\beta \ll 1$

In this case, $c_{2}=\mathcal{O}(\beta)$ hence we put $c_{2}=\beta C$ and the consistency condition (Eq. 5.74) yields

$$
\mathcal{O}\left(\beta^{3}\right)=\beta^{2}[v-(\alpha \nu+\xi) C]^{2},
$$

hence, to leading order, $C=v /(\alpha v+\xi)$. Unfortunately, the resulting value for $c_{2}$ leads to all the leading order terms in the linear Eq. 5.71 for $N_{x}$ to cancel. We thus have to find higher order terms in the expansion for $c_{2}$; due to the form of Eq. 5.75, the next correction term is $\mathcal{O}\left(\beta^{3 / 2}\right)$. Putting $c_{2}=\beta C(1+\widetilde{C} \sqrt{\beta})$, we find

$$
\widetilde{C}^{2}=\frac{\alpha \xi[\alpha \xi \varrho+4 \mu(\alpha \nu+\xi)]}{2 \mu^{2}(\alpha \nu+\xi)^{3}} .
$$

In order to satisfy the inequality (Eq. 5.72), we require the negative root, that is, $\widetilde{C}<0$.

Fig. 13 Graph of the concentrations

$N_{x}, N_{y}, \varrho_{x}, \varrho_{y}, c$ against time on a logarithmic time for the asymptotic limit 2 , with initial conditions $N_{x}=0.2=N_{y}$, $\varrho_{x}=0.45, \varrho_{y}=0.44$, other parameters given by $\alpha=10=\xi, \beta=1=\mu$, $v=0.5, \varrho=2$. Since model equations are in nondimensional form, the time units are arbitrary

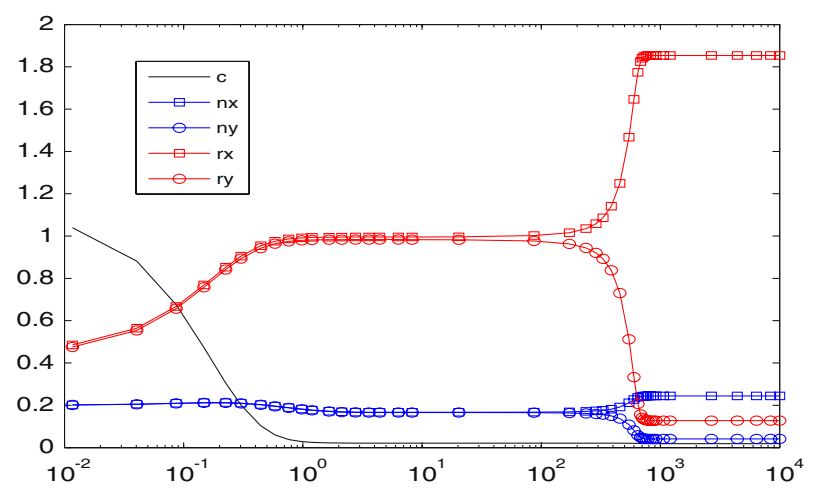


Although the formulae for $N_{x}, N_{y}$ are lengthy, their sum and products simplify to

$$
\Sigma=N_{x}+N_{y}=\frac{\mu \widetilde{C} \sqrt{\beta}(\alpha v+\xi)}{\alpha \xi}, \quad \Pi=N_{x} N_{y}=\frac{\beta \mu}{\alpha \xi} .
$$

The chirality $\phi$ can be simplified using $\phi^{2}=1-4 \Pi / \Sigma^{2}$ which implies

$$
\phi^{2}=\frac{\alpha \varrho \xi-4 \mu(\alpha \nu+\xi)}{\alpha \varrho \xi+4 \mu(\alpha \nu+\xi)} .
$$

Hence we require $\varrho>\varrho_{c}:=4 \mu(\alpha \nu+\xi) / \alpha \xi$ in order for the system to have nonsymmetric steady-states, that is, the system undergoes a symmetry-breaking bifurcation as $\varrho$ increases through $\varrho=\varrho_{c}$. As the mass in the system increases further, the chirality $\phi$ approaches $( \pm)$ unity, indicating a state in which one handedness of crystal completely dominates the other.

\section{Asymptotic Limit 2: $\alpha \sim \xi \gg 1$}

In this case, the left-hand side of the consistency condition (Eq. 5.74) is $\mathcal{O}\left(\alpha^{2} \xi c_{2}^{2}\right)$ whilst the right-hand side is $\mathcal{O}(1)+\mathcal{O}\left(\alpha c_{2}^{2}\right)$, which implies the balance $c_{2}=\mathcal{O}\left(\xi^{-3 / 2}\right)$. Solving for $c_{2}$ leads to

$$
c_{2} \sim \frac{\mu \nu}{\alpha} \sqrt{\frac{2 \beta}{\varrho \xi}}
$$

The leading order equation for $N_{x}, N_{y}$ is then

$$
0=\alpha \xi N^{2}-\alpha N \sqrt{\frac{1}{2} \beta \varrho \xi}+\beta \mu,
$$

hence we find the roots

$$
N_{x}, N_{y} \sim \sqrt{\frac{\beta \varrho}{2 \xi}}, \frac{2 \mu}{\alpha} \sqrt{\frac{\beta}{2 \xi} \varrho}, \quad \varrho_{x}, \varrho_{y} \sim \varrho, \frac{2 \mu}{\alpha} .
$$

Since we have either $\varrho_{x} \gg N_{x} \gg \varrho_{y} \gg N_{y}$ or $\varrho_{y} \gg N_{y} \gg \varrho_{x} \gg N_{x}$, in this asymptotic limit, the system is completely dominated by one species or the other. Putting $\Sigma=N_{x}+N_{y}$ and $\Pi=N_{x} N_{y}$ we have $\phi^{2}=1-4 \Pi / \Sigma^{2} \sim 1-8 \mu / \alpha \varrho$.

\section{Discussion}

We now try to use the above theory and experimental results of Viedma (2005) to estimate the relevant timescales for symmetry-breaking in a prebiotic world. Extrapolating the data of time against grinding rate in rpm from Fig. 2 of Viedma (2005) suggests times of $2 \times 10^{5}$ hours using a straight line fit to $\log$ (time) against $\log (\mathrm{rpm})$ or $1000-3000$ hours if $\log ($ time $)$ against rpm or time against $\log (\mathrm{rpm})$ is fitted. A reduction in the speed of grinding in prebiotic circumstances is expected since natural processes such as water waves are much more likely to operate at the order of a few seconds ${ }^{-1}$ or minutes ${ }^{-1}$ rather than $600 \mathrm{rpm}$.

Similar extrapolations on the number and mass of balls used to much lower amounts gives a further reduction of about 3 , using a linear fit to $\log$ (time) against 
mass of balls from Fig. 1 of Viedma (2005). There is an equally good straight line fit to time against $\log$ (ball-mass) but it is then difficult to know how small a mass of balls would be appropriate in the prebiotic scenario. There is an additional factor due to the experiments of Viedma being on a small volume of $10 \mathrm{ml}$, whereas a sensible volume for prebiotic chemistry is 10001 , giving an additional factor of $10^{5}$. Combining these three factors $\left(10^{3}, 3\right.$, and $\left.10^{5}\right)$ with the 10 days of the original experiment, we estimate that the timescale for prebiotic symmetry breaking is $\mathcal{O}\left(3 \times 10^{9}\right)$ days, which is equivalent to the order of about ten million years.

This extrapolation ignores the time required to arrive at the initial enantiomeric excesses of 5\% used by Viedma (2005) from a small asymmetry caused by either a random fluctuation or by the parity-violation. Although the observed chiral structures are the minimum energy configurations as predicted by parity violation, there is an evens probability that the observed handedness could simply be the result of a random fluctuation which was amplified by the same mechanisms. In order to perform an example calculation, we take a random fluctuation of the size predicted by parity violation, which is of the order of $10^{-17}$, as suggested by Kondepudi and Nelson (1984). Our goal is now to find the time taken to amplify this to an $\mathcal{O}$ (1) $(5 \%)$ enantiomeric excess.

The models derived in this paper, for example in "Asymptotic Limit 2: $\alpha \sim \xi \gg 1$ ", predict that the chiral excess grows exponentially in time. Assuming, from Eq. 5.69, that $\phi\left(t_{0}\right)=10^{-17}$ and $\phi\left(t_{1}\right)=0.1$, then the timescale for the growth of this small perturbation is

$$
t_{1}-t_{0}=\frac{1}{4 \mu \nu} \sqrt{\frac{\xi \varrho}{\beta}} \log \frac{10^{-1}}{10^{-17}}
$$

Since the growth of enantiomeric excess is exponential, it only takes 16 times as long for the perturbation to grow from $10^{-17}$ to $10^{-1}$ as from $10^{-1}$ to 1 . Hence we only need to increase our estimate of the timescale by one power of ten, to 100 million years.

This estimate should be taken as a very rough estimate, since it relies on extrapolating results by many orders of magnitude. Also, given the vast differences in temperature from the putative subzero prebiotic world to a tentative hot hydrothermal vent, there could easily be changes in timescale by a factor of several orders of magnitude.

\section{Conclusions}

After summarising the existing models of chiral symmetry-breaking processes we have systematically derived a model in which through aggregation and fragmentation chiral clusters compete for achiral material. The model is closed, in that there is no input of mass into the system, although the form of the aggregation and fragmentation rate coefficients mean that there is an input of energy, keeping the system away from equilibrium. Furthermore, there is no direct interaction of clusters of opposite handedness; rather just through a simple competition for achiral substrate, the system can spontaneously undergo chiral symmetry-breaking. This model helps explain the experimental results of Viedma (2005) and Noorduin et al. (2008). 
The microscopic model originally derived has been simplified successively to a minimalistic model, which, numerical results show, exhibits symmetry-breaking. Even after this reduction, the model is extremely complex to analyse due to the large number of cluster sizes retained in the model. Hence we construct two truncated models, one truncated at tetramers, which shows no symmetry-breaking and one at hexamers which shows symmetry-breaking under certain conditions on the parameter values. Alternative reductions are proposed: instead of retaining the concentrations of just a few cluster sizes, we retain information about the shape of the distribution, such as the number of clusters and the total mass of material in clusters of each handedness. These reduced models are as simple to analyse as truncated models yet, since they more accurately account for the shape of the size-distribution than a truncated model, are expected to give models which more easily fit to experimental data. Of course, other ansatzes for the shape of the size distributions could be made, and will lead to modified conditions for symmetrybreaking; however, we believe that the qualitative results outlined here will not be contradicted by analyses of other macroscopic reductions.

One noteworthy feature of the results shown herein is that the symmetry-breaking is inherently a product of the two handednesses competing for achiral material. The symmetry-breaking does not rely on critical cluster sizes, which are a common feature of theories of crystallisation, or on complicated arguments about surface area to volume ratios to make the symmetric state unstable. We do not deny that these aspects of crystallisation are genuine, these features are present in the phenomena of crystal growth, but they are not the fundamental cause of chiral symmetrybreaking.

More accurate fitting of the models to experimental data could be acheived if one were to fit the generalised Becker-Döring model (Eqs. 2.11 and 2.12) with realistic rate coefficients. Questions to address include elucidating how the number and size distribution at the start of the grinding influences the end state. For example, if one were to start with a few large right-handed crystals and many small left-handed crystals, would the system convert to entirely left- or entirely right-handed crystals? Answers to these more complex questions may rely on higher moments of the size distributions, surface area to volume ratios and critical cluster nuclei sizes.

\footnotetext{
Acknowledgements I would particularly like to thank Professors Axel Brandenburg and Raphael Plasson for inviting me to an extended programme of study on homochirality at Nordita (Stockholm, Sweden) in February 2008. There I met and benefited greatly from discussions with Professors Meir Lahav, Mike McBride, Wim Noorduin, as well as many others. The models described here are a product of the stimulating discussions held there. I am also grateful for funding under EPSRC springboard fellowship EP/E032362/1.
}

Open Access This article is distributed under the terms of the Creative Commons Attribution Noncommercial License which permits any noncommercial use, distribution, and reproduction in any medium, provided the original author(s) and source are credited.

\section{Appendix A: General Theory for Crystallisation and Grinding with Competition Between Polymorphs}

This model can be generalised so as to be applicable to the case of grinding a system undergoing crystallisation in which several polymorphs of crystal nucleate 
simultaneously. It may then be possible to use grinding to suppress the growth of one polymorph and allow a less stable form to be expressed. In this case, the growth and fragmentation rates of the two polymorphs will differ, we denote the two polymorphs by $x$ and $y$ following Bolton and Wattis (2004). In place of $a, b, \alpha, \xi, \beta$ we have $a_{x, r}$, $a_{y, r}, b_{x, r}, \alpha_{x, r}$, etc. Hence in place of Eqs. 2.20-2.27 we have

$$
\begin{aligned}
\frac{\mathrm{d} x_{r}}{\mathrm{~d} t}= & a_{x, r-1} c_{1} x_{r-1}-b_{x, r} x_{r}-a_{x, r} c_{1} x_{r}+b_{x, r+1} x_{r+1}-\beta_{x, r} x_{r}+\beta_{x, r+2} x_{r+2} \\
& +\left(\alpha_{x, r-2} c_{2}+\xi_{x, r-2} x_{2}\right) x_{r-2}-\left(\alpha_{x, r} c_{2}+\xi_{x, r} x_{2}\right) x_{r}, \quad(r \geq 4) \\
\frac{\mathrm{d} y_{r}}{\mathrm{~d} t}= & a_{y, r-1} c_{1} y_{r-1}-b_{y, r} y_{r}-a_{y, r} c_{1} y_{r}+b_{y, r+1} y_{r+1}-\beta_{y, r} y_{r}+\beta_{y, r+2} y_{r+2} \\
& +\left(\alpha_{y, r-2} c_{2}+\xi_{y, r-2} y_{2}\right) y_{r-2}-\left(\alpha_{y, r} c_{2}+\xi_{y, r} y_{2}\right) y_{r}, \quad(r \geq 4) \\
\frac{\mathrm{d} x_{2}}{\mathrm{~d} t}= & \mu_{x} c_{2}-\mu_{x} v_{x} x_{2}-a_{x, 2} c_{1} x_{2}+b_{x, 3} x_{3}-\left(\alpha_{x, r} c_{2}+\xi_{x, r} x_{2}\right) x_{r} \\
& +\beta_{x, 4} x_{4}+\sum_{k=4}^{\infty} \beta_{x, r} x_{r}-\sum_{k=2}^{\infty} \xi_{x, k} x_{2} x_{k}, \\
\frac{\mathrm{d} y_{2}}{\mathrm{~d} t}= & \mu_{y} c_{2}-\mu_{y} v_{y} y_{2}-a_{y, 2} c_{1} y_{2}+b_{y, 3} y_{3}-\left(\alpha_{y, r} c_{2}+\xi_{y, r} y_{2}\right) y_{r} \\
& +\beta_{y, 4} y_{4}+\sum_{k=4}^{\infty} \beta_{y, r} y_{r}-\sum_{k=2}^{\infty} \xi_{y, k} y_{2} y_{k} \\
\frac{\mathrm{d} c_{1}}{\mathrm{~d} t}= & 2 \epsilon c_{2}-2 \delta c_{1}^{2}-\sum_{k=2}^{\infty}\left(a_{x, k} c_{1} x_{k}-b_{x, k+1} x_{k+1}+a_{y, k} c_{1} y_{k}-b_{y, k+1} y_{k+1}\right) \\
\frac{\mathrm{d} c_{2}}{\mathrm{~d} t}= & a_{x, 2} x_{2} c_{1}-b_{x, 3} x_{3}-a_{x, 3} c_{1} x_{3}+b_{x, 4} x_{4}-\left(\alpha_{x, 3} c_{2}+\xi_{x, 3} x_{2}\right) x_{3}+\beta_{x, 5} x_{5} y_{2}-\left(\mu_{x}+\mu_{y}\right) c_{2}+\delta c_{1}^{2}-\epsilon c_{2}-\sum_{k=2}^{\infty} c_{2}\left(\alpha_{x, r} x_{r}+\alpha_{y, r} y_{r}\right) \\
\frac{\mathrm{d} y_{3}}{\mathrm{~d} t}= & a_{y, 2} y_{2} c_{1}-b_{y, 3} y_{3}-a_{y, 3} c_{1} y_{3}+b_{y, 4} y_{4}-\left(\alpha_{y, 3} c_{2}+\xi_{y, 3} y_{2}\right) y_{3}+\beta_{y, 5} y_{5}
\end{aligned}
$$

For simplicity let us consider an example in which all the growth and fragmentation rate parameters are independent of cluster size, $\left(a_{x, r}=a_{x}, \xi_{y, r}=\xi_{y}\right.$, etc. for all $r$ ). The thermodynamic stability of the two types of crystal depends on their relative interactions with monomers from solution, that is, if $a_{x} / b_{x}>a_{y} / b_{y}$ then $X$ is the more stable form. This is because, in the absence of $c_{2}$, we can define free energy functions

$$
Q_{r}^{x}=\left(\frac{a_{x}}{b_{x}}\right)^{r-1}, \quad Q_{r}^{y}=\left(\frac{a_{y}}{b_{y}}\right)^{r-1}
$$


which generate the equilibrium distributions

$$
c_{r}^{e q x}=Q_{r}^{x} c_{1}^{r}=\frac{b_{x}}{a_{x}}\left(\frac{a_{x} c_{1}}{b_{x}}\right)^{r}>c_{r}^{e q y}=Q_{r}^{y} c_{1}^{r}=\frac{b_{y}}{a_{y}}\left(\frac{a_{y} c_{1}}{b_{y}}\right)^{r} .
$$

If $a_{x} / b_{x}<a_{y} / b_{y}$ then the latter $(Y)$ will be the dominant crystal type at equilibrium, whilst $X$ is the less stable morphology at equilibrium. These last two words are vital, since, at early times, the growth rates depend on the relative sizes of the growth rates $a_{x}$ and $a_{y}$. It is possible for the less stable form to grow first and more quickly from solution, and be observed for a significant period of time, since the rate of convergence to equilibrium also depends on the fragmentation rates and so can be extremely slow (see Wattis 1999 for details).

In the presence of grinding, the crystal size distributions also depend upon the strength of dimer interactions, that is, the growth rates $\alpha_{x} c_{2}+\xi_{x} x_{2}, \alpha_{y} c_{2}+\xi_{y} y_{2}$ and the grinding rates $\beta_{x}, \beta_{y}$. The steady-state size distributions will depend on the relative growth ratios due to grinding $\left(\alpha_{x} c_{2}+\xi_{x} x_{2}\right) / \beta_{x}$ and $\left(\alpha_{y} c_{2}+\xi_{y} y_{2}\right) / \beta_{y}$ as well as the more traditional terms due to growth from solution, namely $a_{x} c_{1} / b_{x}$ and $a_{y} c_{1} / b_{y}$. Such systems with dimer interactions have been analysed previously by Bolton and Wattis (2002). The presence of dimer interactions can alter the size distribution, and in non-symmetric systems such as those analysed here, dimer interactions can alter the two distributions differently. Two points are worth noting here:

(i) for certain parameter values, the less stable stable form ( $Y$, say, with $a_{y} / b_{y}<$ $a_{x} / b_{x}$ ) may be promoted to the more stable morphology by grinding (if ( $\alpha_{y} c_{2}+$ $\left.\xi_{y} y_{2}\right) / \beta_{y}$ is sufficiently greater than $\left.\left(\alpha_{x} c_{2}+\xi_{x} x_{2}\right) / \beta_{x}\right)$;

(ii) grinding may make a less rapidly nucleating and growing form ( $Y$, say, with $a_{y}<a_{x}$ ) into a more rapidly growing form if $\alpha_{y} c_{2}+\xi_{y} y_{2}$ is sufficiently greater than $\alpha_{x} c_{2}+\xi_{2} x_{2}$.

In systems which can crystallise into three or more forms, we may have the case where $x$ is more stable than $y$ and $y$ is more stable than $z$; thus, at equilibrium $x$ will be observed. Furthermore, if $a_{x}<a_{y}>a_{z}$ we may observe type $y$ at early times due to it having faster nucleation and growth rates than $x$ and $z$. However, it is possible that the presence of grinding could suppress both $x$ and $y$ and allow $z$ to be expressed, if some combination of the inequalities

$$
\frac{\alpha_{z} c_{2}+\xi_{z} z_{2}}{\beta_{z}}>\frac{\alpha_{y} c_{2}+\xi_{y} y_{2}}{\beta_{y}}, \frac{\alpha_{x} c_{2}+\xi_{x} x_{2}}{\beta_{x}},
$$

$\alpha_{z}>\alpha_{y}, \alpha_{x}, \xi_{z}>\xi_{x}, \xi_{y}$ hold.

\section{References}

Becker R, Döring W (1935) Kinetische behandlung der keimbildung in übersättigten dämpfen. Ann Phys 24:719-752

Bolton CD, Wattis JAD (2002) Generalised Becker-Döring equations: effect of dimer interactions. J Phys A Math Gen 35:3183-3202

Bolton CD, Wattis JAD (2003) Generalised coarse-grained Becker-Döring equations. J Phys A Math Gen 36:7859-7888

Bolton CD, Wattis JAD (2004) The Becker-Döring equations with input, competition and inhibition. J Phys A Math Gen 37:1971-1986 
Brandenburg A, Andersen AC, Höfner S, Nilsson M (2005a) Homochiral growth through enantiomeric cross-inhibition. Orig Life Evol Biosph 35:225-241. arXiv:q-bio/0401036

Brandenburg A, Andersen AC, Nilsson M (2005b) Dissociation in a polymerization model of homochirality. Orig Life Evol Biosph 35:507-521. arXiv:q-bio/0502008

Coveney PV, Wattis JAD (2006) Coarse-graining and renormalisation group methods for the elucidation of the kinetics of complex nucleation and growth processes. Mol Phys 104:177-185

da Costa FP (1998) Asymptotic behaviour of low density solutions to the generalized Becker-Döring equations. Nonlinear Differ Equ Appl 5:23-37

Darwin C (1887) Private letter to Joseph Hooker (1871). In: Darwin F (ed) The life and letters of Charles Darwin, including an autobiographical chapter, 3 vol, pp 168-169. John Murray, London

Frank FC (1953) On spontaneous asymmetric synthesis. Biochim Biophys Acta 11:459-463

Gleiser M, Walker SI (2008) An extended model for the evolution of prebiotic homochirality: a bottom-up approach to the origin of life. arXiv.org/0802.2884 [q-bio.BM]

Gleiser M, Thorarinson J, Walker SI (2008) Punctuated chirality. arXiv.org/0802.1446 [astro-ph]

Kondepudi DK, Asakura K (2001) Chiral autocatalysis, spontaneous symmetry breaking and stochastic behaviour. Acc Chem Res 34:946-954

Kondepudi DK, Nelson GW (1984) Chiral symmetry breaking in nonequilibrium chemical systems: time scales for chiral selection. Phys Lett A 106:203-206

Kondepudi DK, Nelson GW (1985) Weak neutral currents and the origin of biomolecular chirality. Nature 314:438-441

Kondepudi DK, Kaufman RJ, Singh N (1990) Chiral symmetry-breaking in sodium chlorate crystallization. Science 250:975-976

Kondepudi DK, Bullock KL, Digits JA, Yarborough PD (1995) Stirring rate as a critical parameter in chiral symmetry breaking crystallization. J Am Chem Soc 117:401-404

McBride JM, Tully JC (2008) Did life grind to a start? Nature (News and Views) 452:161-162

Multamaki T, Brandenburg A (2005) Spatial dynamics of homochiralization. Int J Astrobiol 4:73-78. arXiv:q-bio/0505040

Noorduin WL, Izumi T, Millemaggi A, Leeman M, Meekes H, van Enckevort WJP, Kellogg RM, Kaptein B, Vlieg E, Blackmond DG (2008) Emergence of a single solid chiral state from a nearly racemic amino acid derivative. J Am Chem Soc 130:1158-1159

Saito Y, Hyuga H (2004) Complete homochirality induced by the nonlinear autocatalysis and recycling. J Phys Soc Jpn 73:33-35. arXiv:physics/0310142

Saito Y, Hyuga H (2005) Chirality selection in crystallization. J Phys Soc Jpn 74:535-537

Sandars PGH (2003) A toy model for the generation of homochirality during polymerisation. Orig Life Evol Biosph 33:575-583

Soai K, Shibata T, Morioka H, Choji K (1995) Asymmetric autocatalysis and amplification of enantiomeric excess of a chiral molecule. Nature 378:767-768

Uwaha M (2004) A model for complete chiral crystallization. J Phys Soc Jpn 73:2601-2603

Viedma C (2005) Chiral symmetry breaking during crystallization: complete chiral purity induced by nonlinear autocatalysis and recycling. Phys Rev Lett 94:065504

von Smoluchowski M (1916) Drei vorträge über diffusion Brownsche molekular bewegung und koagulation von kolloidteichen. Phys Z 17:557-571

Wattis JAD (1999) A Becker-Döring model of competitive nucleation. J Phys A Math Gen 32: 8755-8784

Wattis JAD, Coveney PV (2005a) Symmetry-breaking in chiral polymerisation. Orig Life Evol Biosph 35:243-273

Wattis JAD, Coveney PV (2005b) Chiral polymerisation and the RNA world. Int J Astronomy 4:63-73 